\title{
Identifying Initial Condition in Degenerate Parabolic Equation with Singular Potential
}

\author{
K. Atifi, Y. Balouki, El-H. Essoufi, and B. Khouiti \\ Laboratoire de Mathématiques, Informatique et Sciences de l'Ingénieur (MISI), Université Hassan 1er, 26000 Settat, Morocco \\ Correspondence should be addressed to B. Khouiti; khouiti_b@yahoo.fr
}

Received 28 September 2016; Revised 13 March 2017; Accepted 19 March 2017; Published 14 June 2017

Academic Editor: Jen-Chih Yao

Copyright (C) 2017 K. Atifi et al. This is an open access article distributed under the Creative Commons Attribution License, which permits unrestricted use, distribution, and reproduction in any medium, provided the original work is properly cited.

\begin{abstract}
A hybrid algorithm and regularization method are proposed, for the first time, to solve the one-dimensional degenerate inverse heat conduction problem to estimate the initial temperature distribution from point measurements. The evolution of the heat is given by a degenerate parabolic equation with singular potential. This problem can be formulated in a least-squares framework, an iterative procedure which minimizes the difference between the given measurements and the value at sensor locations of a reconstructed field. The mathematical model leads to a nonconvex minimization problem. To solve it, we prove the existence of at least one solution of problem and we propose two approaches: the first is based on a Tikhonov regularization, while the second approach is based on a hybrid genetic algorithm (married genetic with descent method type gradient). Some numerical experiments are given.
\end{abstract}

\section{Introduction}

The inverse problem is expressed when the PDE solution is measured or specified, and we are interested in determining some properties: coefficients, forcing term, boundary, or initial condition from the partial knowledge of the system in a limited time interval (see $[1,2])$.

In the last recent years, an increasing interest has been devoted to degenerate parabolic equations. Indeed, many problems coming from physics (boundary layer models in [3], models of Kolmogorov type in [4], etc.), biology (WrightFisher models in [5] and Fleming-Viot models in [6]), and economics (Black-Merton-Scholes equations in [7]) are described by degenerate parabolic equations [8].

The identification of the initial state of nondegenerate parabolic problems is well studied in the literature (see [911]). However, as far as we know, the degenerate case has not been analysed in the literature.

In this paper, we are interested in estimating the initial condition by the variational method in data assimilation of degenerate/singular parabolic equation:

$$
\partial_{t} \psi-\partial_{x}\left(a(x) \partial_{x} \psi(x)\right)-\frac{\lambda}{x^{\beta}} \psi=f, \quad a(x)=x^{\alpha},
$$

where $\Omega=] 0,1[, \alpha \in] 0,1[, \beta \in] 0,2-\alpha[, \lambda \leqslant 0$, and $f \in$ $L^{2}(\Omega \times] 0, T[)$. With initial and boundary conditions

$$
\psi(x, 0)=\psi_{0},\left.\quad \psi\right|_{x=0}=\left.\psi\right|_{x=1}=0 .
$$

The mathematical model leads to a nonconvex minimization problem

$$
\begin{aligned}
\text { find } & \psi_{0} \in A_{\mathrm{ad}} \\
\text { such that } & J\left(\psi_{0}\right)=\min _{u \in A_{\mathrm{ad}}} J(u),
\end{aligned}
$$

where the functional $J$ is defined as follows:

$$
J(u)=\frac{1}{2 T} \int_{0}^{T}\left\|C \psi(t)-\psi^{\mathrm{obs}}(t)\right\|_{L^{2}(\Omega)}^{2},
$$

subject to $\psi$ being the weak solution of the parabolic problem (1) with initial state $u, \psi^{\text {obs }}$ an observation of $\psi$ in $\left.\Omega \times\right] 0, T$, and $C$ the observation operator. The space $A_{\text {ad }}$ is the set of admissible initial states.

Problem (3) is ill-posed in the sense of Hadamard. To solve this problem, we propose two approaches. 
The first approach is based on regularization, for the first time, applied to solve a degenerate inverse problem. The problem thus consists of minimizing a functional of the form

$$
\begin{aligned}
J_{T}(u)= & \frac{1}{2 T} \int_{0}^{T}\left\|C \psi(t)-\psi^{\mathrm{obs}}(t)\right\|_{L^{2}(\Omega)}^{2} d t \\
& +\frac{\varepsilon}{2}\left\|u-\psi^{b}\right\|_{L^{2}(\Omega)}^{2} .
\end{aligned}
$$

Here, the last term in (5) stands for the so-called Tikhonov-type regularization $([12,13]), \varepsilon$ being a small regularizing coefficient that provides extra convexity to the functional $J_{T}$ and $\psi^{b}$ a priori (background) knowledge of the true state $\psi_{0}^{\text {exact }}$ (the state to estimate). We consider that the values of $\psi^{b}$ are given in each point of analysis grid-points.

The second approach is applied when there is a partial knowledge of values of $\psi^{b}$ (example 20\%); the regularization parameter is very difficult to determine. To overcome this problem, we propose a new approach, based on a hybrid genetic algorithm (married genetic with descent method gradient type). Finally, we make a comparison between the two mentioned approaches (with $20 \%$ of $\psi^{b}$ ).

First of all, we prove that problem (3) has at least one solution. The gradient of the functional $J$ is calculated with the adjoint method. Numerical experiments are presented to show the performance of our approaches.

\section{Problem Statement and Main Result}

Consider the following problem:

$$
\begin{aligned}
\partial_{t} \psi+A(\psi) & =f \\
\psi(0, t) & =\psi(1, t)=0 \quad \forall t \in] 0, T[ \\
\psi(x, 0) & =\psi_{0}(x) \quad \forall x \in \Omega,
\end{aligned}
$$

where $\Omega=] 0,1\left[, f \in L^{2}(\Omega \times] 0, T[)\right.$, and $A$ is the operator defined as

$$
A(\psi)=-\partial_{x}\left(a(x) \partial_{x} \psi(x)\right)-\frac{\lambda}{x^{\beta}} \psi, \quad a(x)=x^{\alpha},
$$

with $\alpha \in] 0,1[, \beta \in] 0,2-\alpha[$, and $\lambda \leqslant 0$.

We want to estimate $\psi_{0}$ thanks to an observation $\psi^{\mathrm{obs}}(x, t)$ of $\psi(x, t)$ in $\left.\Omega \times\right] 0, T[$. The minimization problem associated with this problem is

$$
\begin{aligned}
\text { find } & \psi_{0} \in A_{\mathrm{ad}} \\
\text { such that } & J\left(\psi_{0}\right)=\min _{u \in A_{\mathrm{ad}}} J(u),
\end{aligned}
$$

where the functional $J$ is as follows:

$$
J(u)=\frac{1}{2 T} \int_{0}^{T}\left\|C \psi(t)-\psi^{\mathrm{obs}}(t)\right\|_{L^{2}(\Omega)}^{2},
$$

subject to $\psi$ being the weak solution of the parabolic problem (6) with initial state $u, \psi^{b}$ the background state, and $C$ the observation operator. The space $A_{\text {ad }}$ is the set of admissible initial states (will be defined later).

We now specify some notations we shall use. Let us introduce the following functional spaces (see [14-16]):

$$
\begin{aligned}
& V=\{u \\
& \left.\quad \in L^{2}(\Omega), u \text { absolutely continuous on }[0,1]\right\}, \\
& S=\left\{u \in L^{2}(\Omega), \sqrt{a} u_{x} \in L^{2}(\Omega), u(0)=u(1)=0\right\}, \\
& H_{a}^{1}(\Omega)=V \cap S \\
& H_{a}^{2}(\Omega)=\left\{u \in H_{a}^{1}(\Omega), a u_{x} \in H^{1}(\Omega)\right\} \\
& H_{\alpha, 0}^{1}=\left\{u \in H_{\alpha}^{1} \mid u(0)=u(1)=0\right\}, \\
& \left.\left.H_{\alpha}^{1}=\left\{u \in L^{2}(\Omega) \cap H_{\mathrm{Loc}}^{1}(] 0,1\right]\right) \mid x^{\alpha / 2} u_{x} \in L^{2}(\Omega)\right\}
\end{aligned}
$$

with

$$
\begin{aligned}
& \|u\|_{H_{a}^{1}(\Omega)}^{2}=\|u\|_{L^{2}(\Omega)}^{2}+\left\|\sqrt{a} u_{x}\right\|_{L^{2}(\Omega)}^{2}, \\
& \|u\|_{H_{a}^{2}(\Omega)}^{2}=\|u\|_{H_{a}^{1}(\Omega)}^{2}+\left\|\left(a u_{x}\right)_{x}\right\|_{L^{2}(\Omega)}^{2}, \\
& \langle u, v\rangle_{H_{\alpha}^{1}}=\int_{\Omega}\left(u v+x^{\alpha} u_{x} v_{x}\right) d x .
\end{aligned}
$$

We recall that (see [16]) $H_{a}^{1}$ is an Hilbert space and it is the closure of $C_{c}^{\infty}(0,1)$ for the norm $\|\cdot\|_{H_{a}^{1}}$. If $1 / \sqrt{a} \in L^{1}(\Omega)$ then the injections

$$
\begin{aligned}
& H_{a}^{1}(\Omega) \hookrightarrow L^{2}(\Omega) \\
& H_{a}^{2}(\Omega) \hookrightarrow H_{a}^{1}(\Omega) \\
& H^{1}\left(0, T ; L^{2}(\Omega)\right) \cap L^{2}(0, T ; D(A)) \\
& \quad \hookrightarrow L^{2}\left(0, T ; H_{a}^{1}\right) \cap C\left(0, T ; L^{2}(\Omega)\right)
\end{aligned}
$$

are compacts.

Firstly, we prove that problem (6) is well-posed, the functional $J$ is continuous, and $J$ is $G$-derivable in $A_{\text {ad }}$.

The weak formulation of problem (6) is

$$
\begin{gathered}
\int_{\Omega} \partial_{t} \psi v d x+\int_{\Omega}\left(a(x) \partial_{x} \psi \partial_{x} v-\frac{\lambda}{x^{\beta}} \psi v\right) d x \\
=\int_{\Omega} f v d x, \quad \forall v \in H_{0}^{1}(\Omega) .
\end{gathered}
$$

Let

$$
B[\psi, v]=\int_{\Omega}\left(a(x) \partial_{x} \psi \partial_{x} v-\frac{\lambda}{x^{\beta}} \psi v\right) d x
$$

We discuss the following cases.

(1) Noncoercive Case (see [14], $\lambda=0$ ). In this case, the bilinear form $B$ becomes

$$
B[\psi, v]=\int_{\Omega}\left(a(x) \partial_{x} \psi \partial_{x} v\right) d x
$$


We have $a(x)=0$ at $x=0$, from where the bilinear form $B$ will be noncoercive.

Let

$$
A_{\mathrm{ad}}=\left\{u \in H_{a}^{1}(\Omega) ;\|u\|_{H_{a}^{1}(\Omega)} \leqslant r\right\},
$$

where $r$ is a real strictly positive constant.

We recall the following theorem.

Theorem 1 (see $[14,17,18])$. For all $f \in L^{2}(\Omega \times] 0, T[)$ and $\psi_{0} \in L^{2}(0,1)$, there exists a unique weak solution which solves problem (6) such that

$$
\psi \in C^{0}\left([0, T] ; L^{2}(\Omega)\right) \cap L^{2}\left(0, T ; H_{a}^{1}\right)
$$

and there is a constant $C_{T}$ such that for any solution of (6)

$$
\begin{gathered}
\sup _{t \in[0, T]}\|\psi(t)\|_{L^{2}(\Omega)}^{2}+\int_{0}^{T}\left\|\sqrt{a} \psi_{x}(t)\right\|_{L^{2}(\Omega)}^{2} d t \\
\leq C_{T}\left(\left\|\psi_{0}\right\|_{L^{2}(\Omega)}^{2}+\|f\|_{\left.\left.L^{2}(\Omega \times] 0, T\right]\right)}^{2}\right) ;
\end{gathered}
$$

if more $\psi_{0} \in H_{a}^{1}(\Omega)$ then

$$
\begin{gathered}
\psi \in C^{0}\left([0 ; T], H_{a}^{1}\right) \cap L^{2}\left(0, T ; H_{a}^{2}\right) \\
\cap H^{1}\left(0, T ; L^{2}(\Omega)\right)
\end{gathered}
$$

and there is a constant $C_{T}$ such that

$$
\begin{aligned}
& \sup _{t \in[0, T]}\|\psi(t)\|_{H_{a}^{1}}^{2} \\
& \quad+\int_{0}^{T}\left(\left\|\psi_{t}\right\|_{L^{2}(\Omega)}^{2}+\left\|\left(a \psi_{x}\right)_{x}(t)\right\|_{L^{2}(\Omega)}^{2}\right) d t \\
& \quad \leqslant C_{T}\left(\left\|\psi_{0}\right\|_{H_{a}^{1}}^{2}+\|f\|_{\left.L^{2}(\Omega \times] 0, T\right]}^{2}\right) .
\end{aligned}
$$

Theorem 2. Let $\psi$ be the weak solution of (6) with initial state $\psi_{0}$. In noncoercive case, the function

$$
\begin{aligned}
\varphi: H_{a}^{1}(\Omega) \longrightarrow & C^{0}\left([0, T] ; H_{a}^{1}(\Omega)\right) \\
& \cap L^{2}\left(0, T ; H_{a}^{2}(\Omega)\right) \\
& \cap H^{1}\left(0, T ; L^{2}(\Omega)\right) \\
\psi_{0} \longmapsto & \psi
\end{aligned}
$$

is continuous, and the functional $J$ has at least one minimum in $A_{\text {ad }}$.

Theorem 3. Let $\psi$ be the weak solution of (6) with initial state $\psi_{0}$. The function

$$
\begin{aligned}
\varphi: H_{a}^{1}(\Omega) \longrightarrow & C^{0}\left([0, T] ; H_{a}^{1}(\Omega)\right) \\
& \cap L^{2}\left(0, T ; H_{a}^{2}(\Omega)\right) \\
& \cap H^{1}\left(0, T ; L^{2}(\Omega)\right) \\
\psi_{0} \longmapsto & \psi
\end{aligned}
$$

is G-derivable in $A_{a d}$.
(2) Subcritical Potential Case (see $[19,20], \lambda \neq 0$ ). Then the bilinear form $B$ becomes

$$
B[\psi, v]=\int_{\Omega}\left(a(x) \partial_{x} \psi \partial_{x} v-\frac{\lambda}{x^{\beta}} \psi v\right) d x .
$$

Since $a(x)=0$ at $x=0$ and $\lim _{x \rightarrow 0}\left(\lambda / x^{\beta}\right)=+\infty$, the bilinear form $B$ is noncoercive and is noncontinuous at $x=0$.

Consider the not bounded operator $(K, D(K))$ where

$$
\begin{aligned}
& K u=\left(x^{\alpha} u_{x}\right)_{x}+\frac{\lambda}{x^{\beta}} u, \quad \forall u \in D(K), \\
& \left.D(k)=\left\{u \in H_{\alpha, 0}^{1} \cap H_{\text {Loc }}^{2}(] 0,1\right]\right) \mid\left(x^{\alpha} u_{x}\right)_{x}+\frac{\lambda}{x^{\beta}} u \\
& \left.\quad \in L^{2}(\Omega)\right\} .
\end{aligned}
$$

Let

$$
A_{\mathrm{ad}}=\left\{u \in L^{2}(\Omega) ;\|u\|_{L^{2}(\Omega)} \leqslant r\right\}
$$

where $r$ is a real strictly positive constant.

We recall the following theorem.

Theorem 4 (see $[15,19])$. If $f=0$ then, for all $\psi_{0} \in L^{2}(\Omega)$, problem (6) has a unique weak solution

$$
\begin{gathered}
\left.\left.\psi \in C^{0}\left([0, T] ; L^{2}(\Omega)\right) \cap C^{0}(] 0, T\right] ; D(K)\right) \\
\left.\left.\cap C^{1}(] 0, T\right] ; L^{2}(\Omega)\right)
\end{gathered}
$$

if more $\psi_{0} \in D(K)$ then

$$
\psi \in C^{0}([0, T] ; D(K)) \cap C^{1}\left([0, T] ; L^{2}(\Omega)\right) ;
$$

if $f \in L^{2}(] 0,1[\times] 0, T[)$ then, for all $\psi_{0} \in L^{2}(\Omega)$, problem (6) has a unique solution

$$
\psi \in C^{0}\left([0, T] ; L^{2}(\Omega)\right) .
$$

Theorem 5. Let $\psi$ be the weak solution of (6) with initial state $\psi_{0}$. In subcritical potential case, the function

$$
\begin{aligned}
\varphi: L^{2}(\Omega) & \longrightarrow C\left([0, T] ; L^{2}(\Omega)\right) \\
\psi_{0} & \longmapsto \psi
\end{aligned}
$$

is continuous, and the functional $J$ is continuous in $A_{\text {ad }}$.

Theorem 6. Let $\psi$ be the weak solution of (6) with initial state $\psi_{0}$. The function

$$
\begin{aligned}
\varphi: L^{2}(\Omega) & \longrightarrow C\left([0, T] ; L^{2}(\Omega)\right) \\
\psi_{0} & \longmapsto \psi
\end{aligned}
$$

is G-derivable in $A_{a d}$. 


\section{Proof}

Proof of Theorem 2. Let $\delta \psi_{0} \in H_{a}^{1}(\Omega)$ be a small variation such that $\psi_{0}+\delta \psi_{0} \in A_{\mathrm{ad}}$.

Consider $\delta \psi=\psi^{\delta}-\psi$, with $\psi$ being the weak solution of (6) with initial state $\psi_{0}$ and $\psi^{\delta}$ is the weak solution of (6) with initial state $\psi_{0}^{\delta}=\psi_{0}+\delta \psi_{0}$. lem:

Consequently, $\delta \psi$ is the solution of the variational prob-

$$
\begin{aligned}
& \int_{\Omega} \partial_{t} \delta \psi v d x+\int_{\Omega} a(x) \partial_{x} \delta \psi(x) \partial_{x} v d x=0 \\
& \delta \psi(0, t)=\delta \psi(1, t)=0 \quad \forall t \in] 0, T[ \\
& \delta \psi(x, 0)=\delta \psi_{0}(x) \quad \forall x \in \Omega .
\end{aligned}
$$

Hence, $\delta \psi$ is the weak solution of (6) with $f=0$. We apply the estimate in Theorem 1 with $f=0$. This gives the following.

There is a constant $C_{T}$ such that

$$
\begin{aligned}
& \sup _{t \in[0, T]}\|\delta \psi(t)\|_{H_{a}^{1}(\Omega)}^{2} \\
& \quad+\int_{0}^{T}\left(\left\|\partial_{t} \delta \psi\right\|_{L^{2}(\Omega)}^{2}+\left\|\partial_{x}\left(a \partial_{x} \delta \psi\right)(t)\right\|_{L^{2}(\Omega)}^{2}\right) d t \\
& \leqslant C_{T}\left\|\delta \psi_{0}\right\|_{H_{a}^{1}(\Omega)}^{2} ;
\end{aligned}
$$

therefore

$$
\begin{gathered}
\sup _{t \in[0, T]}\|\delta \psi(t)\|_{H_{a}^{1}(\Omega)}^{2} \leqslant C_{T}\left\|\delta \psi_{0}\right\|_{H_{a}^{1}(\Omega)}^{2} \\
\|\delta \psi\|_{C\left([0, T] ; H_{a}^{1}(\Omega)\right)}^{2} \leqslant C_{T}\left\|\delta \psi_{0}\right\|_{H_{a}^{1}(\Omega)}^{2} .
\end{gathered}
$$

And from (32) we have

$$
\begin{aligned}
& \|\delta \psi(t)\|_{H_{a}^{1}(\Omega)}^{2}+\int_{0}^{T}\left\|\partial_{x}\left(a \partial_{x} \delta \psi\right)(t)\right\|_{L^{2}(\Omega)}^{2} d t \\
& \leqslant C_{T}\left\|\delta \psi_{0}\right\|_{H_{a}^{1}(\Omega)}^{2}, \quad \forall t \in[0, T] \\
& \int_{0}^{T}\|\delta \psi(t)\|_{H_{a}^{1}(\Omega)}^{2} d t+T \int_{0}^{T}\left\|\partial_{x}\left(a \partial_{x} \delta \psi\right)(t)\right\|_{L^{2}(\Omega)}^{2} d t \\
& \quad \leqslant T C_{T}\left\|\delta \psi_{0}\right\|_{H_{a}^{1}(\Omega)}^{2} \\
& \inf (1, T)\left(\int_{0}^{T}\|\delta \psi(t)\|_{H_{a}^{1}(\Omega)}^{2} d t\right. \\
& \left.\quad+\int_{0}^{T}\left\|\partial_{x}\left(a \partial_{x} \delta \psi\right)(t)\right\|_{L^{2}(\Omega)}^{2} d t\right) \leqslant T C_{T}\left\|\delta \psi_{0}\right\|_{H_{a}^{1}(\Omega)}^{2} \\
& \int_{0}^{T}\|\delta \psi(t)\|_{H_{a}^{1}(\Omega)}^{2} d t+\int_{0}^{T}\left\|\partial_{x}\left(a \partial_{x} \delta \psi\right)(t)\right\|_{L^{2}(\Omega)}^{2} d t \\
& \quad \leqslant \frac{T C_{T}}{\inf (1, T)}\left\|\delta \psi_{0}\right\|_{H_{a}^{1}(\Omega)}^{2} \cdot
\end{aligned}
$$

Hence,

$$
\|\delta \psi\|_{L^{2}\left(0, T, H_{a}^{2}(\Omega)\right)}^{2} \leqslant \frac{T C_{T}}{\inf (1, T)}\left\|\delta \psi_{0}\right\|_{H_{a}^{1}(\Omega)}^{2} .
$$

In addition, from (32) we have

$$
\begin{aligned}
& \|\delta \psi(t)\|_{H_{a}^{1}(\Omega)}^{2} \\
& +\int_{0}^{T}\left\|\partial_{t} \delta \psi(t)\right\|_{L^{2}(\Omega)}^{2} d t \leqslant C_{T}\left\|\delta \psi_{0}\right\|_{H_{a}^{1}(\Omega)}^{2}, \\
& \forall t \in[0, T]
\end{aligned}
$$

$$
\begin{aligned}
& \|\delta \psi(t)\|_{L^{2}(\Omega)}^{2}+\left\|\sqrt{a} \partial_{x} \delta \psi(t)\right\|_{L^{2}(\Omega)}^{2} \\
& \quad+\int_{0}^{T}\left\|\partial_{t} \delta \psi(t)\right\|_{L^{2}(\Omega)}^{2} d t \leqslant C_{T}\left\|\delta \psi_{0}\right\|_{H_{a}^{1}(\Omega)}^{2}
\end{aligned}
$$

$\forall t \in[0, T]$

$$
\begin{aligned}
& \|\delta \psi(t)\|_{L^{2}(\Omega)}^{2} \\
& \quad+\int_{0}^{T}\left\|\partial_{t} \delta \psi(t)\right\|_{L^{2}(\Omega)}^{2} d t \leqslant C_{T}\left\|\delta \psi_{0}\right\|_{H_{a}^{1}(\Omega)}^{2},
\end{aligned}
$$

$$
\forall t \in[0, T]
$$

$$
\begin{aligned}
& \int_{0}^{T}\|\delta \psi(t)\|_{L^{2}(\Omega)}^{2} d t \\
& \quad+T \int_{0}^{T}\left\|\partial_{t} \delta \psi(t)\right\|_{L^{2}(\Omega)}^{2} d t \leqslant T C_{T}\left\|\delta \psi_{0}\right\|_{H_{a}^{1}(\Omega)}^{2} \\
& \|\delta \psi\|_{H^{1}\left(0, T ; L^{2}(\Omega)\right)}^{2} \leqslant \frac{T C_{T}}{\inf (1, T)}\left\|\delta \psi_{0}\right\|_{H_{a}^{1}(\Omega)}^{2} .
\end{aligned}
$$

Equations (34), (36), and (41) imply the continuity of the function

$$
\begin{aligned}
\varphi: H_{a}^{1}(\Omega) \longrightarrow & C^{0}\left([0, T] ; H_{a}^{1}(\Omega)\right) \\
& \cap L^{2}\left(0, T ; H_{a}^{2}(\Omega)\right) \\
& \cap H^{1}\left(0, T ; L^{2}(\Omega)\right) \\
\psi_{0} \longmapsto & \psi .
\end{aligned}
$$

Hence, the functional $J$ is continuous in

$$
A_{\mathrm{ad}}=\left\{u \in H_{a}^{1}(\Omega) ;\|u\|_{H_{a}^{1}(\Omega)} \leqslant r\right\}
$$

We have $1 / \sqrt{a(x)}=x^{-\alpha / 2} \in L^{1}(0,1)$, where $\alpha \epsilon$ ]0,1 [, which gives $H_{a}^{1}(\Omega) \underset{\text { compact }}{\hookrightarrow} L^{2}(\Omega)$. Since the set $A_{\mathrm{ad}}$ is bounded in $H_{a}^{1}(\Omega)$, then $A_{\mathrm{ad}}$ is a compact in $L^{2}(\Omega)$. Therefore, $J$ has at least one minimum in $A_{\mathrm{ad}}$. 
Proof of Theorem 3. Let $\psi_{0} \in A_{\mathrm{ad}}$ and $\delta \psi_{0}$ such that $\psi_{0}+\delta \psi_{0} \in$ $A_{\text {ad }}$; we define the function

$$
\varphi^{\prime}\left(\psi_{0}\right): \delta \psi_{0} \in A_{\mathrm{ad}} \longrightarrow \delta \psi
$$

where $\delta \psi$ is the solution of the variational problem

$$
\begin{aligned}
& \int_{\Omega} \partial_{t}(\delta \psi) v d x+\int_{\Omega} a(x) \partial_{x}(\delta \psi) \partial_{x} v d x=0 \\
& \forall v \in H_{0}^{1}(\Omega)
\end{aligned}
$$

$$
\begin{aligned}
& \delta \psi(0, t)=\delta \psi(1, t)=0 \quad \forall t \in] 0, T[ \\
& \delta \psi(x, 0)=\delta \psi_{0} \quad \forall x \in \Omega
\end{aligned}
$$

and we pose

$$
\phi\left(\psi_{0}\right)=\varphi\left(\psi_{0}+\delta \psi_{0}\right)-\varphi\left(\psi_{0}\right)-\varphi^{\prime}\left(\psi_{0}\right) \delta \psi_{0}
$$

We want to show that

$$
\phi\left(\psi_{0}\right)=o\left(\delta \psi_{0}\right)
$$

We easily verify that the function $\phi$ is solution of the following variational problem:

$$
\begin{aligned}
& \int_{\Omega} \partial_{t} \phi v d x+\int_{\Omega} a(x) \partial_{x} \phi \partial_{x} v d x=0 \quad \forall v \in H_{0}^{1}(\Omega) \\
& \phi(0, t)=\phi(1, t)=0 \\
&\forall t \in] 0, T[ \\
& \phi(x, 0)=\delta \psi_{0}-\left(\delta \psi_{0}\right)^{2} \\
& \forall x \in \Omega .
\end{aligned}
$$

By the same way as that used in the proof of continuity, we deduce

$$
\begin{aligned}
\|\phi\|_{C\left([0, T], H_{a}^{1}(\Omega)\right)}^{2} & \leqslant C_{T}\left\|\delta \psi_{0}-\left(\delta \psi_{0}\right)^{2}\right\|_{H_{a}^{1}(\Omega)}^{2}, \\
\|\phi\|_{L^{2}\left(0, T, H_{a}^{2}(\Omega)\right)}^{2} & \leqslant \frac{T C_{T}}{\inf (1, T)}\left\|\delta \psi_{0}-\left(\delta \psi_{0}\right)^{2}\right\|_{H_{a}^{1}(\Omega)}^{2}, \\
\|\phi\|_{H^{1}\left(0, T ; L^{2}(\Omega)\right)}^{2} & \leqslant \frac{T C_{T}}{\inf (1, T)}\left\|\psi_{0}-\left(\delta \psi_{0}\right)^{2}\right\|_{H_{a}^{1}(\Omega)}^{2} .
\end{aligned}
$$

Hence, the function $\varphi: \psi_{0} \rightarrow \psi$ is $G$-derivable in $A_{\text {ad }}$ and we deduce the existence of the gradient of the functional $J$.

Proof of Theorem 5. Let $\delta \psi_{0} \in L^{2}(\Omega)$ be a small variation such that $\psi_{0}+\delta \psi_{0} \in A_{\mathrm{ad}}$.

Consider $\delta \psi=\psi^{\delta}-\psi$, with $\psi$ being the weak solution of (6) with initial state $\psi_{0}$, and $\psi^{\delta}$ is the weak solution of (6) with initial state $\psi_{0}^{\delta}=\psi_{0}+\delta \psi_{0}$.
Consequently, $\delta \psi$ is the solution of variational problem

$$
\begin{array}{r}
\int_{\Omega} \partial_{t} \delta \psi v d x+\int_{\Omega}\left(a(x) \partial_{x} \delta \psi \partial_{x} v-\frac{\lambda}{x^{\beta}} \delta \psi v\right) d x=0, \\
\forall v \in H_{0}^{1}(\Omega)
\end{array}
$$

$\delta \psi(0, t)=\delta \psi(1, t)=0 \quad \forall t \in] 0, T[$

$\delta \psi(x, 0)=\delta \psi_{0}(x) \quad \forall x \in \Omega$.

Take $v=\delta \psi$; this gives

$$
\begin{aligned}
& \int_{\Omega} \partial_{t} \delta \psi \delta \psi d x+\int_{\Omega}\left(a(x)\left(\partial_{x} \delta \psi\right)^{2}-\frac{\lambda}{x^{\beta}}(\delta \psi)^{2}\right) d x \\
& \quad=0
\end{aligned}
$$

since $\Omega$ is independent of $t$, which gives

$$
\begin{aligned}
& \frac{1}{2} \frac{d}{d t} \int_{\Omega}(\delta \psi)^{2} d x d t \\
& \quad+\int_{\Omega}\left(a(x)\left(\partial_{x} \delta \psi\right)^{2}-\frac{\lambda}{x^{\beta}}(\delta \psi)^{2}\right) d x=0 .
\end{aligned}
$$

By integrating between 0 and $t$ with $t \in[0, T]$ we obtain

$$
\begin{aligned}
& \frac{1}{2}\|\delta \psi(t)\|_{L^{2}(\Omega)}^{2} \\
& \quad+\int_{0}^{t} \int_{\Omega}\left(a(x)\left(\partial_{x} \delta \psi\right)^{2}-\frac{\lambda}{x^{\beta}}(\delta \psi)^{2}\right) d x d t \\
& \quad=\frac{1}{2}\|\delta \psi(0)\|_{L^{2}(\Omega)}^{2} \\
& \frac{1}{2}\|\delta \psi(t)\|_{L^{2}(\Omega)}^{2} \\
& \quad+\int_{0}^{t} \int_{\Omega}\left(a(x)\left(\partial_{x} \delta \psi\right)^{2}-\frac{\lambda}{x^{\beta}}(\delta \psi)^{2}\right) d x d t \\
& \quad=\frac{1}{2}\left\|\delta \psi_{0}\right\|_{L^{2}(\Omega)}^{2},
\end{aligned}
$$

and since $a(x) \geqslant 0$ and $-\lambda / x^{\beta}>0, \forall x \in \Omega$, we obtain

$$
\|\delta \psi(t)\|_{L^{2}(\Omega)} \leqslant\left\|\delta \psi_{0}\right\|_{L^{2}(\Omega)}
$$

this gives

$$
\operatorname{Sup}_{t \in[0, T]}\|\delta \psi(t)\|_{L^{2}(\Omega)} \leq\left\|\delta \psi_{0}\right\|_{L^{2}(\Omega)} .
$$

From where

$$
\|\delta \psi(t)\|_{C\left([0 ; T], L^{2}(\Omega)\right)} \leq\left\|\delta \psi_{0}\right\|_{L^{2}(\Omega)} .
$$

Which gives the continuity of the function

$$
\begin{aligned}
& \varphi: L^{2}(\Omega) \longrightarrow C\left([0, T] ; L^{2}(\Omega)\right) \\
& \psi_{0} \longmapsto \psi .
\end{aligned}
$$


Hence, the functional $J$ is continuous in

$$
A_{\mathrm{ad}}=\left\{u \in L^{2}(\Omega) ;\|u\|_{L^{2}(\Omega)} \leqslant r\right\} .
$$

Proof of Theorem 6. Let $\psi_{0} \in A_{\mathrm{ad}}$ and $\delta \psi_{0}$ such that $\psi_{0}+\delta \psi_{0} \in$ $A_{\text {ad }}$; we define the function

$$
\varphi^{\prime}\left(\psi_{0}\right): \delta \psi_{0} \in A_{\mathrm{ad}} \longrightarrow \delta \psi,
$$

where $\delta \psi$ is the solution of the variational problem

$$
\begin{aligned}
& \int_{\Omega} \partial_{t}(\delta \psi) v d x \\
& +\int_{\Omega}\left(a(x) \partial_{x}(\delta \psi) \partial_{x} v-\frac{\lambda}{x^{\beta}} \delta \psi v\right) d x=0
\end{aligned}
$$

$$
\forall v \in H_{0}^{1}(\Omega)
$$

$$
\begin{aligned}
& \delta \psi(0, t)=\delta \psi(1, t)=0 \quad \forall t \in] 0, T[ \\
& \delta \psi(x, 0)=\delta \psi_{0} \quad \forall x \in \Omega
\end{aligned}
$$

and we pose

$$
\phi\left(\psi_{0}\right)=\varphi\left(\psi_{0}+\delta \psi_{0}\right)-\varphi\left(\psi_{0}\right)-\varphi^{\prime}\left(\psi_{0}\right) \delta \psi_{0}
$$

We want to show that

$$
\phi\left(\psi_{0}\right)=o\left(\delta \psi_{0}\right)
$$

We easily verify that the function $\phi$ is the solution of the following variational problem:

$$
\begin{aligned}
& \int_{\Omega} \partial_{t} \phi v d x+\int_{\Omega}\left(a(x) \partial_{x} \phi \partial_{x} v-\frac{\lambda}{x^{\beta}} \phi v\right) d x=0 \\
& \forall v \in H_{0}^{1}(\Omega) \\
& \phi(0, t)=\phi(1, t)=0 \quad \forall t \in] 0, T[ \\
& \phi(x, 0)=\delta \psi_{0}-\left(\delta \psi_{0}\right)^{2} \quad \forall x \in \Omega .
\end{aligned}
$$

By the same way as that used in the proof of continuity, we deduce

$$
\|\phi\|_{C\left([0, T], L^{2}(\Omega)\right)} \leqslant\left\|\delta \psi_{0}-\left(\delta \psi_{0}\right)^{2}\right\|_{L^{2}(\Omega)} .
$$

Hence, in all cases, the function $\varphi: \psi_{0} \rightarrow \psi$ is G-derivable in $A_{\mathrm{ad}}$ and we deduce the existence of the gradient of the functional $J$.

Now, we are going to compute the gradient of $J$ with the adjoint state method.

\section{Gradient of $J$}

We define the Gâteaux derivative of $\psi$ at $\psi_{0}$ in the direction $h \in L^{2}(\Omega)$, by

$$
\widehat{\psi}=\lim _{\varepsilon \rightarrow 0} \frac{\psi\left(\psi_{0}+\varepsilon h\right)-\psi\left(\psi_{0}\right)}{\varepsilon},
$$

where $\psi\left(\psi_{0}+\varepsilon h\right)$ is the weak solution of (6) with initial state $\psi_{0}+\varepsilon h$, and $\psi\left(\psi_{0}\right)$ is the weak solution of (6) with initial state $\psi_{0}$.

We compute the Gâteaux (directional) derivative of (6) at $\psi_{0}$ in some direction $h \in L^{2}(\Omega)$, and we get the so-called tangent linear model:

$$
\begin{aligned}
\partial_{t} \widehat{\psi}+A \widehat{\psi} & =0 \\
\widehat{\psi}(0, t) & =\widehat{\psi}(1, t)=0 \quad \forall t \in] 0, T[ \\
\widehat{\psi}(x, 0) & =h \quad \forall x \in \Omega .
\end{aligned}
$$

We introduce the adjoint variable $P$, and we integrate

$$
\begin{aligned}
& \int_{0}^{1} \int_{0}^{T} \partial_{t} \widehat{\psi} P d t d x+\int_{0}^{1} \int_{0}^{T} A \widehat{\psi} P d x=0 \\
& \int_{0}^{1}\left([\widehat{\psi} P]_{0}^{T}-\int_{0}^{T} \widehat{\psi} \partial_{t} P d t\right) d x \\
& \quad+\int_{0}^{T}\langle A \widehat{\psi}, P\rangle_{L^{2}(\Omega)} d t=0 \\
& \int_{0}^{1}[\widehat{\psi}(T) P(T)-\widehat{\psi}(0) P(0)] d x \\
& \quad-\int_{0}^{T}\left\langle\widehat{\psi}, \partial_{t} P\right\rangle_{L^{2}(\Omega)} d t+\int_{0}^{T}\langle A \widehat{\psi}, P\rangle_{L^{2}(\Omega)} d t=0 .
\end{aligned}
$$

Let us take $P(x=0)=P(x=1)=0$; then we may write $\langle\widehat{\psi}, A P\rangle_{L^{2}(\Omega)}=\langle A \widehat{\psi}, P\rangle_{L^{2}(\Omega)}$.

And with $P(T)=0$ we may now rewrite (69) as

$$
\int_{0}^{1} \widehat{\psi}(0) P(0) d x+\int_{0}^{T}\left\langle\widehat{\psi}, \partial_{t} P-A P\right\rangle_{L^{2}(\Omega)} d t=0
$$

this gives

$$
\begin{aligned}
\int_{0}^{T}\left\langle\widehat{\psi}, \partial_{t} P-A P\right\rangle_{L^{2}(\Omega)} d t & =\langle-P(0), h\rangle_{L^{2}(\Omega)} \\
P(x=0) & =P(x=1)=0, \\
P(T) & =0 .
\end{aligned}
$$

The Gâteaux derivative of the functional

$$
J\left(\psi_{0}\right)=\frac{1}{2 T} \int_{0}^{T}\left\|C \psi(t)-\psi^{\mathrm{obs}}(t)\right\|_{L^{2}(\Omega)}^{2} d t
$$

at $\psi_{0}$ in the direction $h \in L^{2}(\Omega)$ is given by

$$
\widehat{J}(h)=\lim _{\varepsilon \rightarrow 0} \frac{J\left(\psi_{0}+\varepsilon h\right)-J\left(\psi_{0}\right)}{\varepsilon} .
$$


After some calculations, we arrive at

$$
\widehat{J}(h)=\frac{1}{T} \int_{0}^{T}\left\langle C^{*}\left(C \psi-\psi^{\mathrm{obs}}\right), \widehat{\psi}\right\rangle_{L^{2}(\Omega)} d t .
$$

The adjoint model is

$$
\begin{aligned}
\partial_{t} P-A P & =\frac{1}{T} C^{*}\left(C \psi-\psi^{\mathrm{obs}}\right) \\
P(x=0) & =P(x=1)=0 \quad \forall t \in] 0, T[ \\
P(T) & =0 .
\end{aligned}
$$

Problem (75) is retrograde; we make the change of variable $t \leftrightarrow T-t$, which gives

$$
\begin{aligned}
\partial_{t} P+A P & =\frac{1}{T} C^{*}\left(\widetilde{\psi}^{\mathrm{obs}}-C \widetilde{\psi}\right) \\
P(x=0) & =P(x=1)=0 \quad \forall t \in] 0, T[ \\
P(0) & =0,
\end{aligned}
$$

with $\widetilde{\psi}(t)=\psi(T-t)$.

From (71), (74), and (75) the gradient of $J$ is given by

$$
\frac{\partial J}{\partial \psi_{0}}=-P(0) \text {. }
$$

With the change of variable $t \leftrightarrow T-t$, the gradient becomes

$$
\frac{\partial J}{\partial \psi_{0}}=-P(T) .
$$

To calculate a gradient of $J$, we solve two problems: (6) and (76). The result solution of (6) is used in the second member of problem (76).

\section{Discretization of Problem}

Step 1 (full discretization). To resolve problem (6) and (76), we use the method $\theta$-schema in time. This method is unconditionally stable for $1>\theta \geq 1 / 2$.

Let $h$ be the steps in space and $\Delta t$ the steps in time.

Let

$$
\begin{aligned}
x_{i} & =i h, \quad i \in\{0,1,2, \ldots, N+1\}, \\
c\left(x_{i}\right) & =a\left(x_{i}\right), \\
t_{j} & =j \Delta t, \quad j \in\{0,1,2, \ldots, M+1\}, \\
f_{i}^{j} & =f\left(x_{i}, t_{j}\right) ;
\end{aligned}
$$

we put

$$
\psi_{i}^{j}=\psi\left(x_{i}, t_{j}\right)
$$

Let

$$
\begin{aligned}
d a\left(x_{i}\right) & =\frac{c\left(x_{i+1}\right)-c\left(x_{i}\right)}{h}, \\
b(x) & =-\frac{\lambda}{x^{\beta}} .
\end{aligned}
$$

Therefore,

$$
\partial_{t} \psi+A \psi=f
$$

is approximated by

$$
\begin{aligned}
& -\frac{\theta \Delta t}{h^{2}} c\left(x_{i}\right) \psi_{i-1}^{j+1}+\left(1+\frac{2 \theta \Delta t}{h^{2}} c\left(x_{i}\right)+d a\left(x_{i}\right) \frac{\theta \Delta t}{h}\right. \\
& \left.+b\left(x_{i}\right) \theta \Delta t\right) \psi_{i}^{j+1}-\left(\frac{\theta \Delta t}{h^{2}} c\left(x_{i}\right)+d a\left(x_{i}\right) \frac{\theta \Delta t}{h}\right) \\
& \cdot \psi_{i+1}^{j+1}=\frac{(1-\theta) \Delta t}{h^{2}} c\left(x_{i}\right) \psi_{i-1}^{j}+(1 \\
& -\frac{(1-\theta) \Delta t}{h} d a\left(x_{i}\right)-\frac{2(1-\theta) \Delta t}{h^{2}} c\left(x_{i}\right) \\
& \left.-(1-\theta) b\left(x_{i}\right) \Delta t\right) \psi_{i}^{j}+\left(\frac{(1-\theta) \Delta t}{h} d a\left(x_{i}\right)\right. \\
& \left.+\frac{(1-\theta) \Delta t}{h^{2}} c\left(x_{i}\right)\right) \psi_{i+1}^{j}+\Delta t \cdot\left[(1-\theta) f_{i}^{j}\right. \\
& \left.+\theta f_{i}^{j+1}\right] .
\end{aligned}
$$

Let us define

$$
\begin{aligned}
g_{1}\left(x_{i}\right)= & -\frac{\theta \Delta t}{h^{2}} c\left(x_{i}\right), \\
g_{2}\left(x_{i}\right)= & 1+\frac{2 \theta \Delta t}{h^{2}} c\left(x_{i}\right)+d a\left(x_{i}\right) \frac{\theta \Delta t}{h}+b\left(x_{i}\right) \theta \Delta t, \\
g_{3}\left(x_{i}\right)= & -\frac{\theta \Delta t}{h^{2}} c\left(x_{i}\right)-d a\left(x_{i}\right) \frac{\theta \Delta t}{h}, \\
k_{1}\left(x_{i}\right)= & \frac{(1-\theta) \Delta t}{h^{2}} c\left(x_{i}\right), \\
k_{2}\left(x_{i}\right)= & 1-\frac{(1-\theta) \Delta t}{h} d a\left(x_{i}\right)-\frac{2(1-\theta) \Delta t}{h^{2}} c\left(x_{i}\right) \\
& -(1-\theta) b\left(x_{i}\right) \Delta t, \\
k_{3}\left(x_{i}\right)= & \frac{(1-\theta) \Delta t}{h} d a\left(x_{i}\right)+\frac{(1-\theta) \Delta t}{h^{2}} c\left(x_{i}\right) .
\end{aligned}
$$

$$
\begin{aligned}
D \psi^{j+1} & =B \psi^{j}+V^{j} \text { avec } j \in\{1,2, \ldots, M\} \\
\psi^{0} & =\left(\psi_{0}(i h)\right)_{i \in\{1,2, \ldots, N\}},
\end{aligned}
$$


where

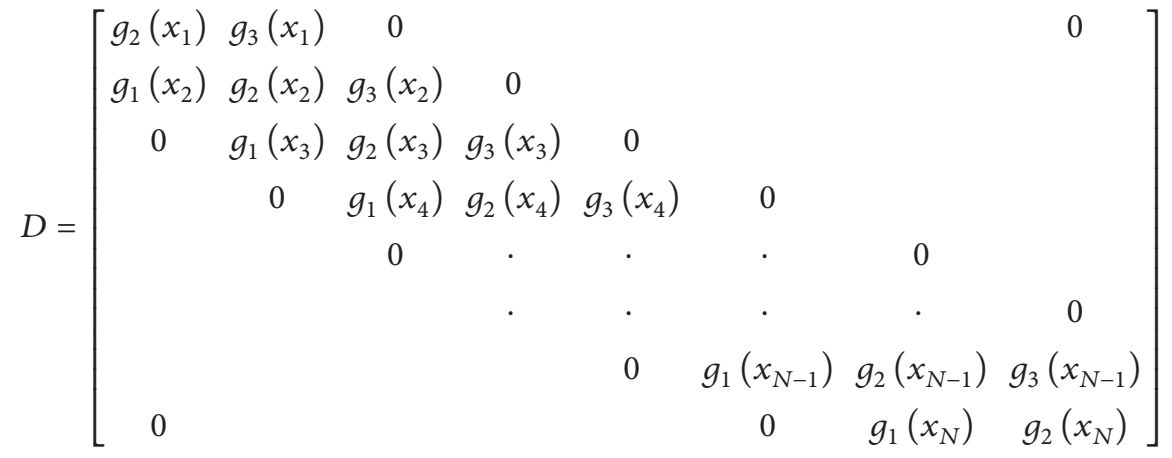

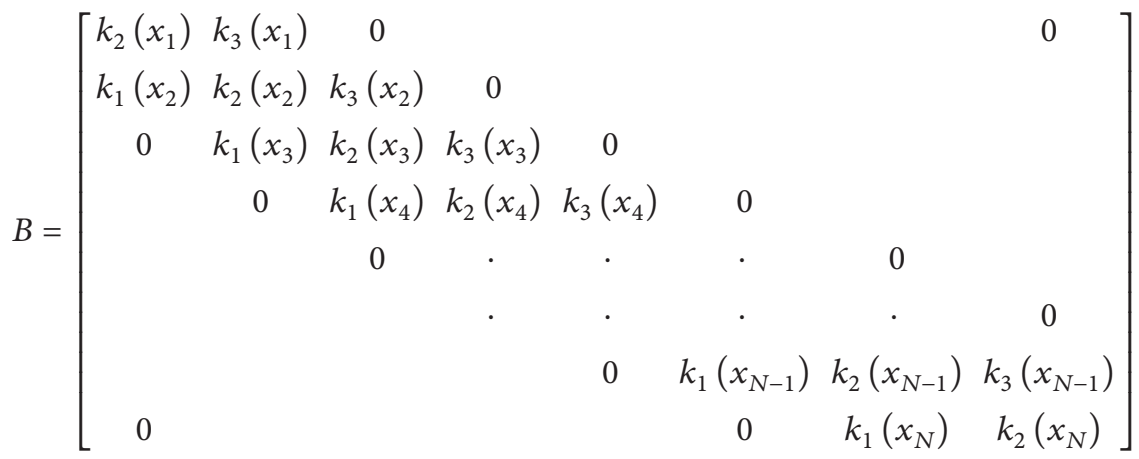

$$
\begin{aligned}
& V^{j}=\left[\begin{array}{c}
\Delta t \cdot\left[(1-\theta) f\left(x_{1}, t_{j}\right)+\theta f\left(x_{1}, t_{j}+\Delta t\right)\right] \\
\Delta t \cdot\left[(1-\theta) f\left(x_{2}, t_{j}\right)+\theta f\left(x_{2}, t_{j}+\Delta t\right)\right] \\
\cdot \\
\cdot \\
\cdot \\
\cdot \\
\Delta t \cdot\left[(1-\theta) f\left(x_{N-1}, t_{j}\right)+\theta f\left(x_{N-1}, t_{j}+\Delta t\right)\right] \\
\Delta t \cdot\left[(1-\theta) f\left(x_{N}, t_{j}\right)+\theta f\left(x_{N}, t_{j}+\Delta t\right)\right]
\end{array}\right] .
\end{aligned}
$$

Step 2 (discretization of the functional one has).

$$
J(u)=\frac{1}{2 T} \int_{0}^{T} \int_{0}^{1}\left(C \psi(x, t)-\psi^{\mathrm{obs}}(x, t)\right)^{2} d x d t .
$$

We recall that the method of Thomas Simpson to calculate an integral is

$$
\begin{aligned}
& \int_{a}^{b} f(x) d x \simeq \frac{h}{2}\left[f\left(x_{0}\right)+2 \sum_{i=1}^{(N+1) / 2-1} f\left(x_{2 i}\right)\right. \\
& \left.+4 \sum_{i=1}^{(N+1) / 2} f\left(x_{2 i+1}\right)+f\left(x_{N+1}\right)\right]
\end{aligned}
$$

with $x_{0}=a, x_{N+1}=b, x_{i}=a+i h, i \in\{1, \ldots, N+1\}$.
Let the functions

$$
\begin{aligned}
\varphi(x, t) & =\left(C \psi(x, t)-\psi^{\mathrm{obs}}(x, t)\right)^{2} \\
\forall t \in[0, T], x \in \Omega, & \\
\varnothing(t) & =\int_{0}^{1} \varphi(x, t) d x .
\end{aligned}
$$

This gives

$$
\begin{gathered}
\varnothing(t) \simeq \frac{h}{2}\left[\varphi(0, t)+2 \sum_{i=1}^{(N+1) / 2-1} \varphi\left(x_{2 i}, t\right)\right. \\
\left.+4 \sum_{i=1}^{(N+1) / 2} \varphi\left(x_{2 i+1}, t\right)+\varphi(1, t)\right],
\end{gathered}
$$


where

$$
\begin{aligned}
\int_{0}^{T} \int_{0}^{1}\left(C \psi(x, t)-\psi^{\mathrm{obs}}(x, t)\right)^{2} d x d t \simeq \int_{0}^{T} \varnothing(t) d t \\
\simeq \frac{d t}{2}\left[\varnothing(0)+2 \sum_{j=1}^{(M+1) / 2-1} \varnothing\left(t_{2 j}\right)\right. \\
\left.\quad+4 \sum_{j=1}^{(M+1) / 2} \varnothing\left(t_{2 j+1}\right)+\varnothing(T)\right],
\end{aligned}
$$

with $t_{0}=0, t_{M+1}=T, t_{j}=j d t, j \in\{1, \ldots, M+1\}$.

Therefore,

$$
\begin{aligned}
J(u) & \simeq \frac{1}{2 T} \frac{d t}{2}\left[\varnothing(0)+2 \sum_{j=1}^{(M+1) / 2-1} \varnothing\left(t_{2 j}\right)\right. \\
& \left.+4 \sum_{j=1}^{(M+1) / 2} \varnothing\left(t_{2 j+1}\right)+\varnothing(T)\right] .
\end{aligned}
$$

Step 3 (discretization of $\nabla J$ ). The adjoint problem (76) is discretized as (85), so,

$$
\nabla J \simeq-P^{M+1} .
$$

\section{Numerical Experiments and Results}

In this section, we discuss two cases:

In case we have a priori knowledge $\psi^{b}$ of $\psi_{0}^{\text {exact }}$ in each point of analysis grid-points, we apply the Tikhonov approach to solve the minimization problem (8). The data $\psi^{b}$ is assumed to be corrupted by measurement errors, which we will refer to as noise. In particular, we suppose that $\psi^{b}=$ $\psi_{0}^{\text {exact }}+e$. Here, we study the impact of err $\left(\operatorname{err}=\|e\|_{2}\right)$ on the construction of the solution.

In case we have a partial knowledge of values of $\psi^{b}$ (example 20\%): firstly, we apply the hybrid approach to rebuild the initial state. Secondly, we make a comparison between both hybrid and Tikhonov approaches.

The tests have been performed in Matlab 2012A, on a Windows 7 platform.

6.1. Regularization Approach. The differentiability and continuity in $A_{\text {ad }}$ of the functional,

$$
\begin{aligned}
J_{T}\left(\psi_{0}\right)= & \frac{1}{2 T} \int_{0}^{T}\left\|C \psi(t)-\psi^{\mathrm{obs}}(t)\right\|_{L^{2}(\Omega)}^{2} d t \\
& +\frac{\varepsilon}{2}\left\|\psi_{0}-\psi^{b}\right\|_{L^{2}(\Omega)}^{2},
\end{aligned}
$$

is deduced from the differentiability and continuity of the functional $J$, and we have

$$
\frac{\partial J_{T}}{\partial \psi_{0}}=-P(T)+\varepsilon\left(\psi_{0}-\psi^{b}\right),
$$

where $P$ is the solution of (76).

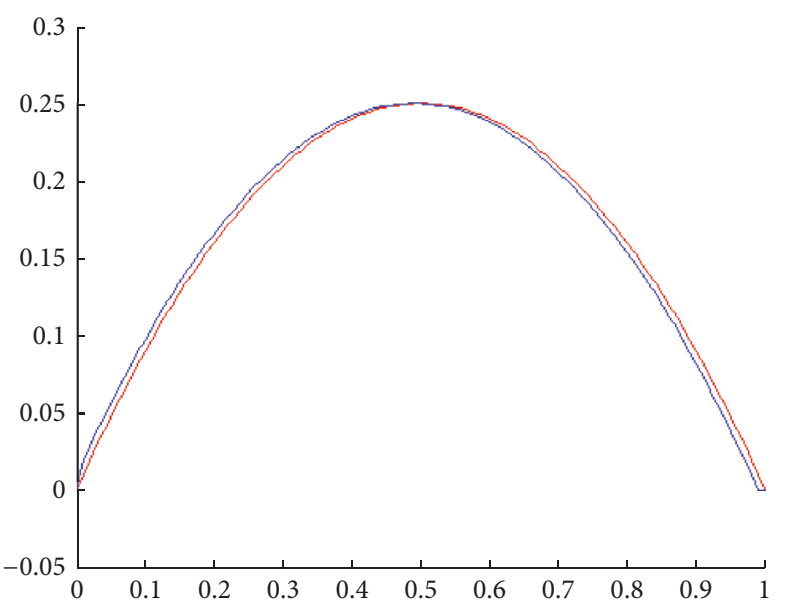

FIgURE 1: Initial temperature. This figure shows that we can rebuild the initial state.

The main steps for descent method at each iteration are the following:

(i) Calculate $\psi^{k}$ solution of (6) with initial condition $\psi_{0}$.

(ii) Calculate $P^{k}$ solution of (76).

(iii) Calculate the descent direction $d_{k}=-\nabla J_{T}\left(\psi_{0}\right)$.

(iv) Find $t_{k}=\operatorname{argmin}_{t>0} J_{T}\left(\psi_{0}+t d_{k}\right)$

(v) Update the variable $\psi_{0}=\psi_{0}+t_{k} d_{k}$.

The algorithm ends when $\left|J_{T}\left(\psi_{0}\right)\right|<\mu$, where $\mu$ is a given small precision.

$t_{k}$ is chosen by the inaccurate linear search by Rule Armijo-Goldstein as follows:

$$
\begin{aligned}
& \text { let } \alpha_{i}, \beta \in[0,1[\text { and } \alpha>0 \\
& \text { if } J_{T}\left(\psi_{0}^{k}+\alpha_{i} d_{k}\right) \leqslant J_{T}\left(\psi_{0}^{k}\right)+\beta \alpha_{i} d_{k}^{T} d_{k} \\
& t_{k}=\alpha_{i} \text { and stop } \\
& \text { if not } \\
& \alpha_{i}=\alpha \alpha_{i} .
\end{aligned}
$$

We do all the tests on Pc with the following configurations: Intel Core i3 CPU 2.27 GHz; RAM = 4 GB (2.93 usable).

In all figures, the observed function is drawn in red and built function in blue.

Let $N$ be number of points in space and $M$ number of points in time.

6.1.1. The Noncoercive Case. Let $\alpha=1 / 2, \lambda=0$ and parameters $N=100, M=100$.

(i) Tests with err $=0$. See Figures 1, 2, 3, and 4 .

(ii) Tests with err $\neq 0$. In Figures 5, 6, 7, and 8, $\psi_{0}^{\text {exact }}$ is drawn in red and $\psi_{0}$ (rebuilt initial condition) in blue.

6.1.2. Sub Critical Potential Case. Let $\alpha=1 / 2, \lambda=-(1-\alpha)^{2} / 4$, $\beta=3 / 4$ and the parameters $N=100, M=100$. 

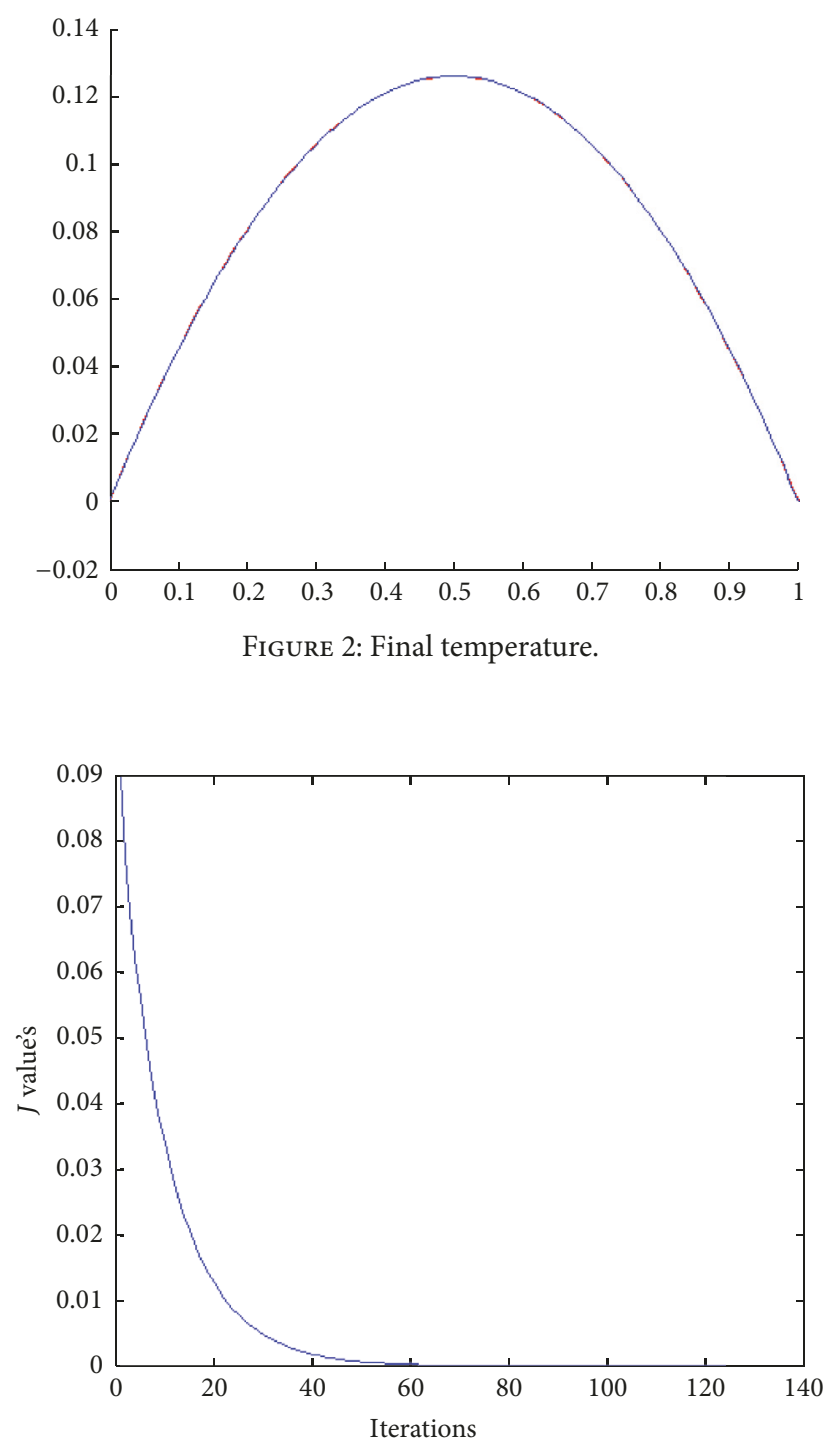

Figure 3: Graph of $J$.

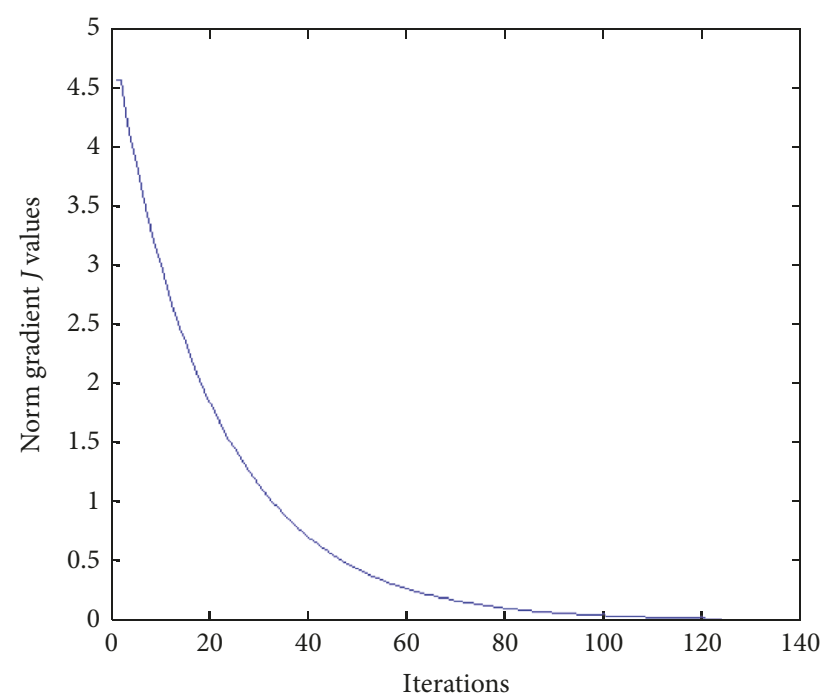

Figure 4: Norm of gradient of $J$.

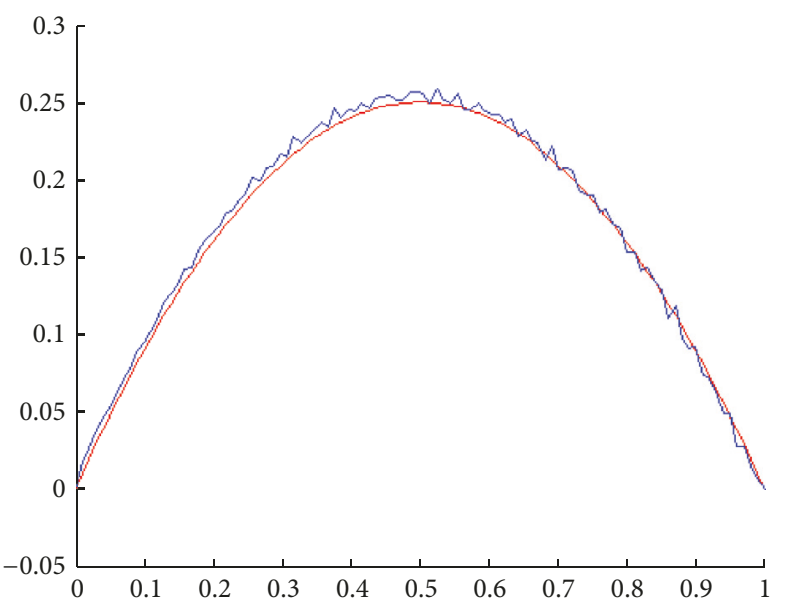

FIGURE 5: Initial temperature in err $=2 \%\left\|\psi_{0}^{\text {exact }}\right\|_{2}$ case. This figure shows that we can rebuild the true state.

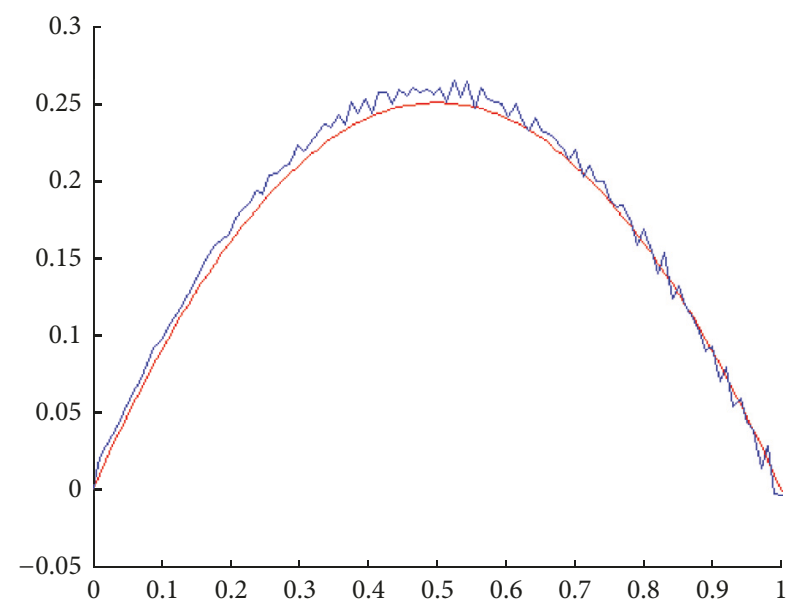

FIGURE 6: Initial temperature in err $=5 \%\left\|\psi_{0}^{\text {exact }}\right\|_{2}$ case. The reconstructed initial condition is not far from the true state.

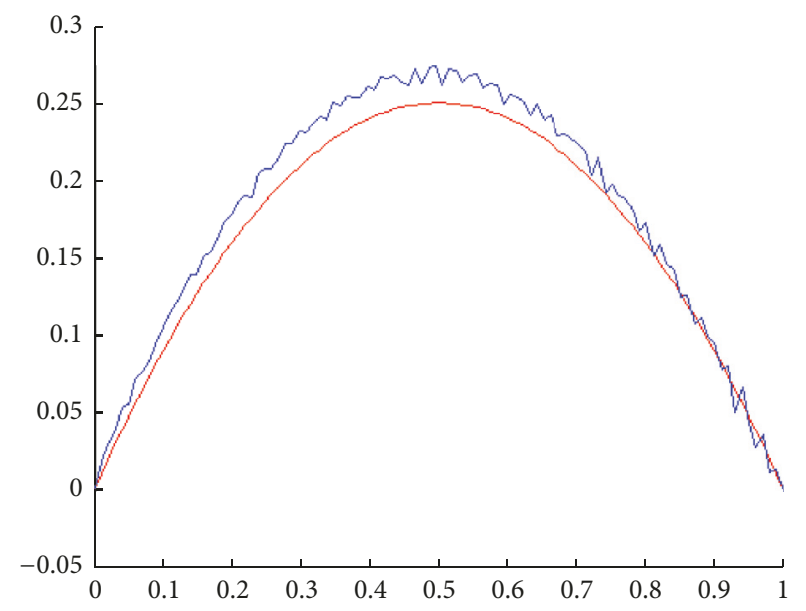

FIGURE 7: Initial temperature in err $=10 \%\left\|\psi_{0}^{\text {exact }}\right\|_{2}$ case. The reconstructed initial condition begins to move away from the true state. 


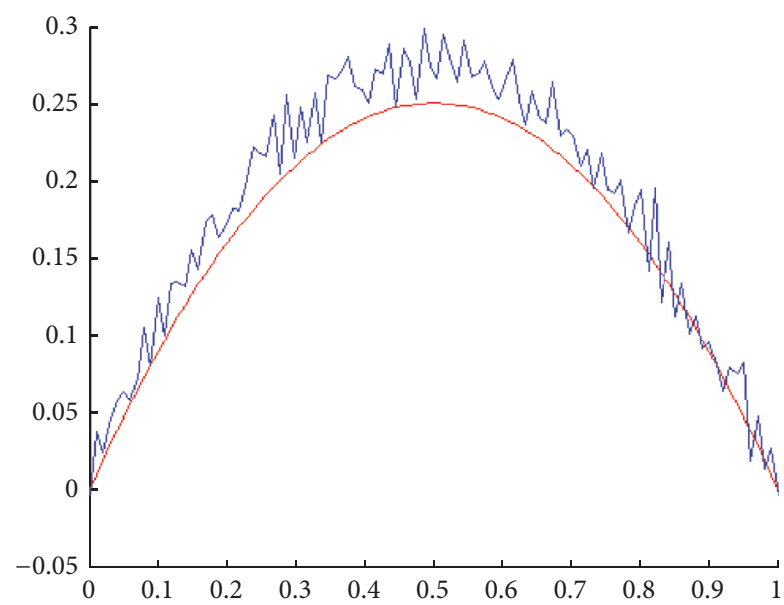

FIGURE 8: Initial temperature in err $=20 \%\left\|\psi_{0}^{\text {exact }}\right\|_{2}$ case. This figure shows that we cannot rebuild the true state.

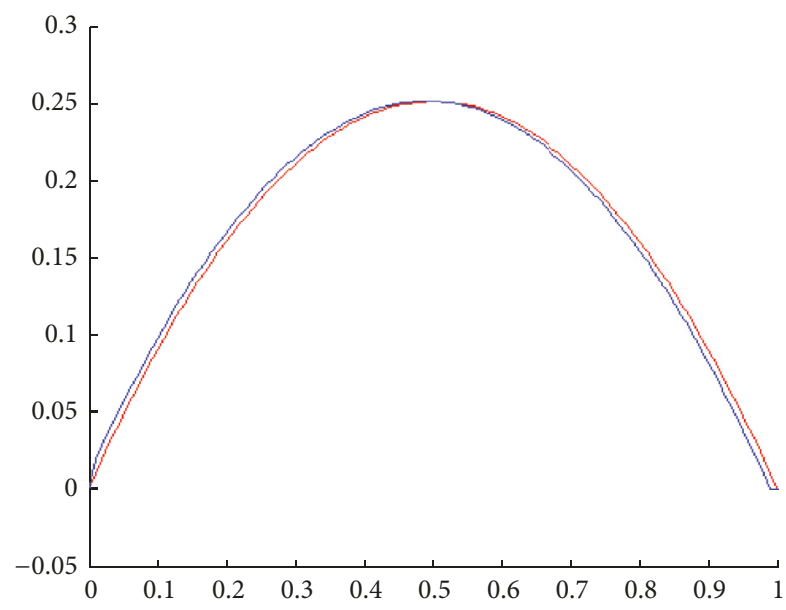

FIGURE 9: Initial temperature. This figure shows that we can rebuild the initial state.

(i) Tests with err $=0$. See Figures 9, 10, 11, and 12.

(ii) Tests with err $\neq 0$. See Figures 13, 14, 15, and 16.

6.2. Hybrid Algorithm. The genetic algorithms (GA) are adaptive search and optimization methods that are based on the genetic processes of biological organisms. Their principles have been first laid down by Holland. The aim of GA is to optimize a problem-defined function, called the fitness function. To do this, GA maintain a population of individuals (suitably represented candidate solutions) and evolve this population over time. At each iteration, called generation, the new population is created by the process of selecting individuals according to their level of fitness in the problem domain and breeding them together using operators borrowed from natural genetics, as, for instance, crossover and mutation. As the population evolves, the individuals in general tend toward

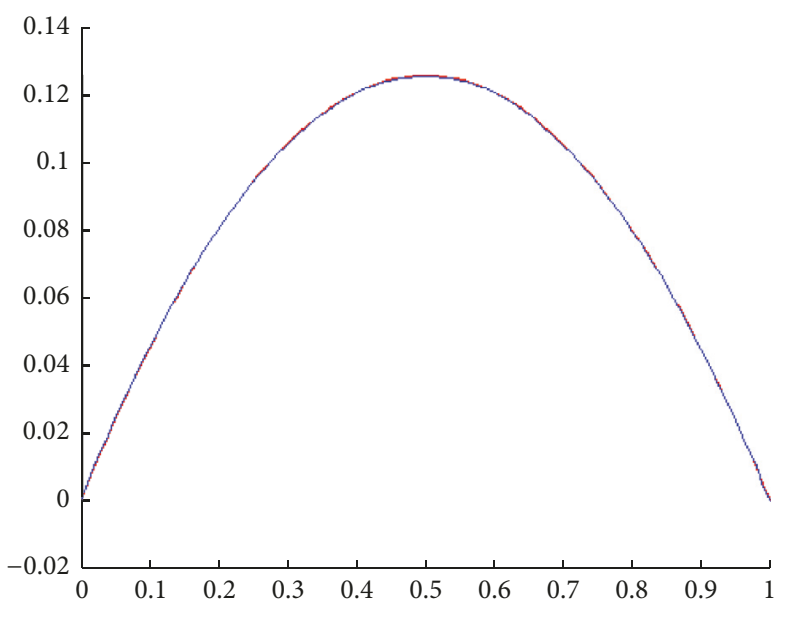

FIGURE 10: Final temperature.

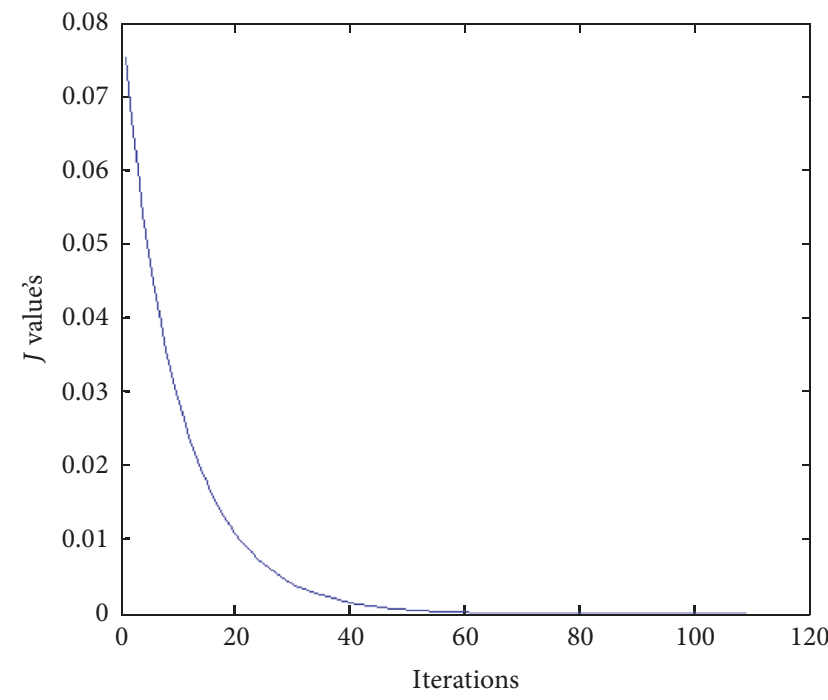

FIGURE 11: Graph of $J$.

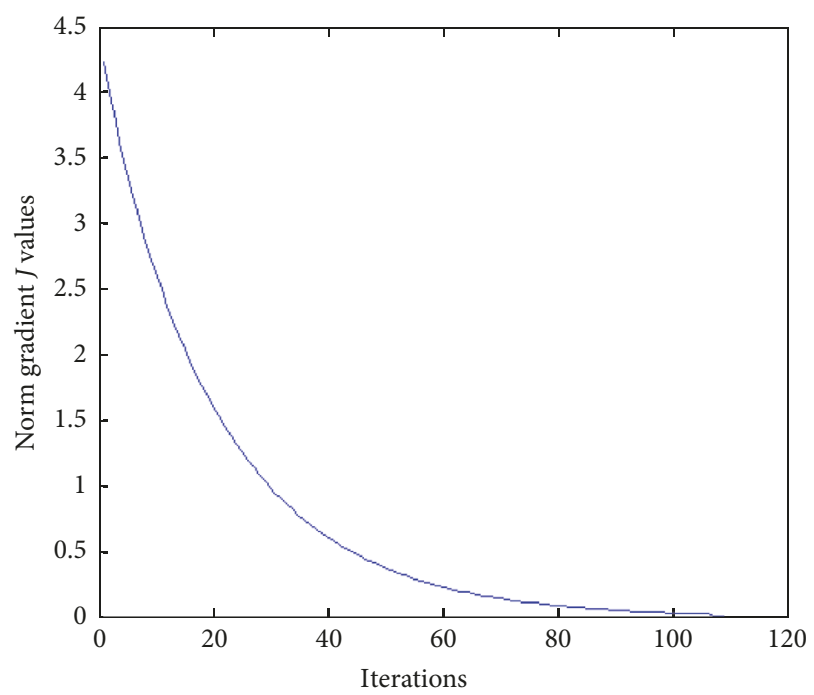

FIGURE 12: Norm of gradient of $J$. 


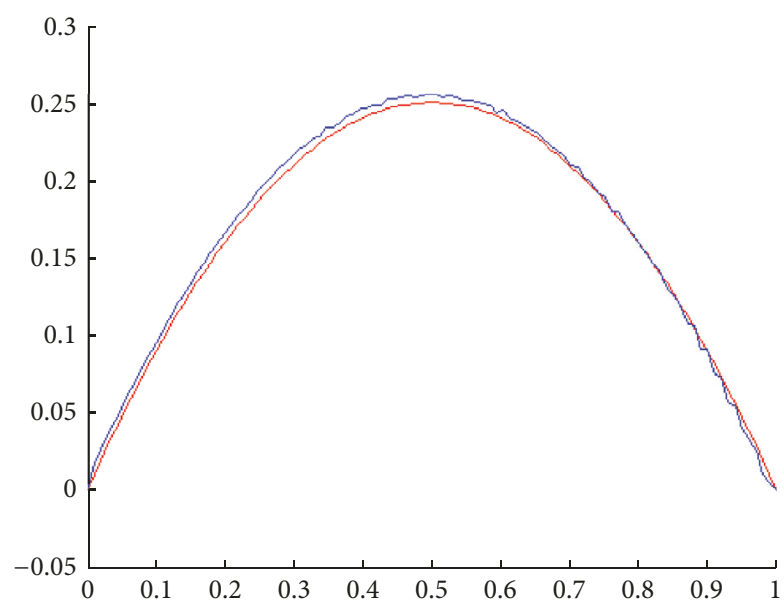

FIgURE 13: Initial temperature in err $=2 \%\left\|\psi_{0}^{\text {exact }}\right\|_{2}$ case. This figure shows that we can rebuild the true state.

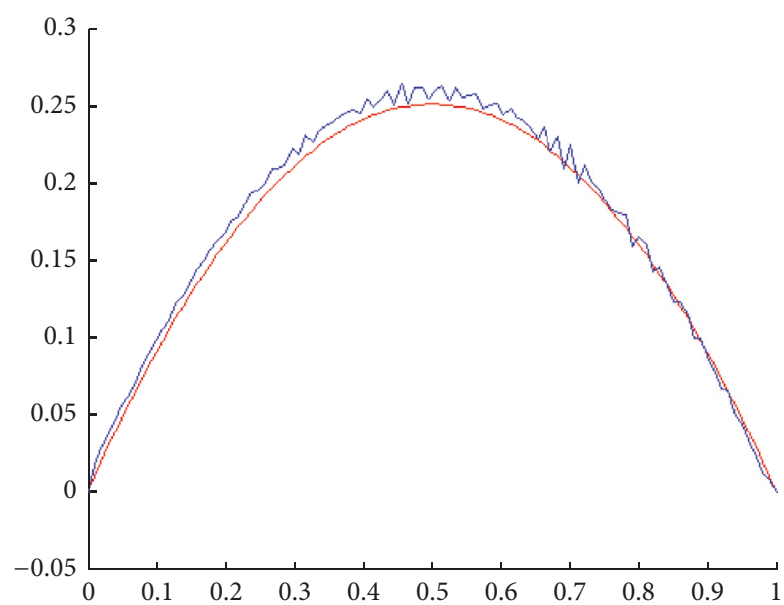

Figure 14: Initial temperature in err $=5 \%\left\|\psi_{0}^{\text {exact }}\right\|_{2}$ case. The reconstructed initial condition is not far from the true state.

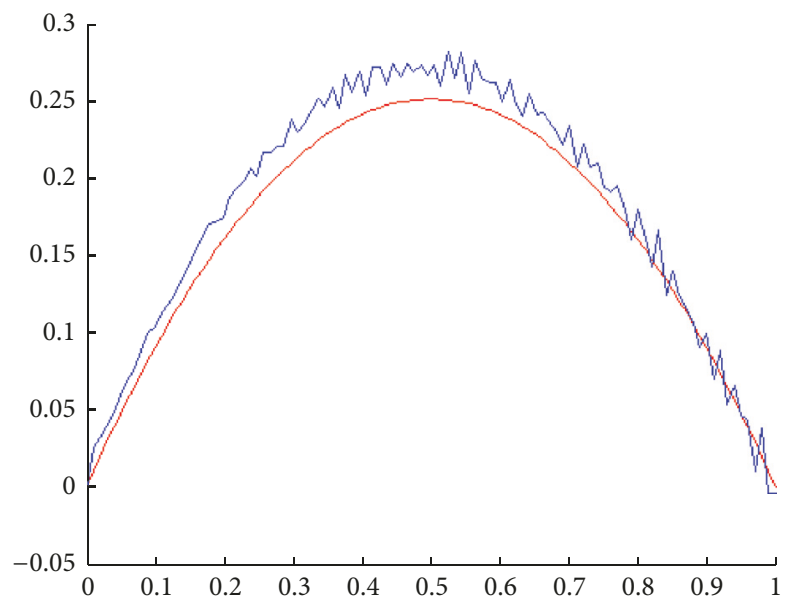

FIGURE 15: Initial temperature in err $=10 \%\left\|\psi_{0}^{\text {exact }}\right\|_{2}$ case. The reconstructed initial condition began to move away from the true state.

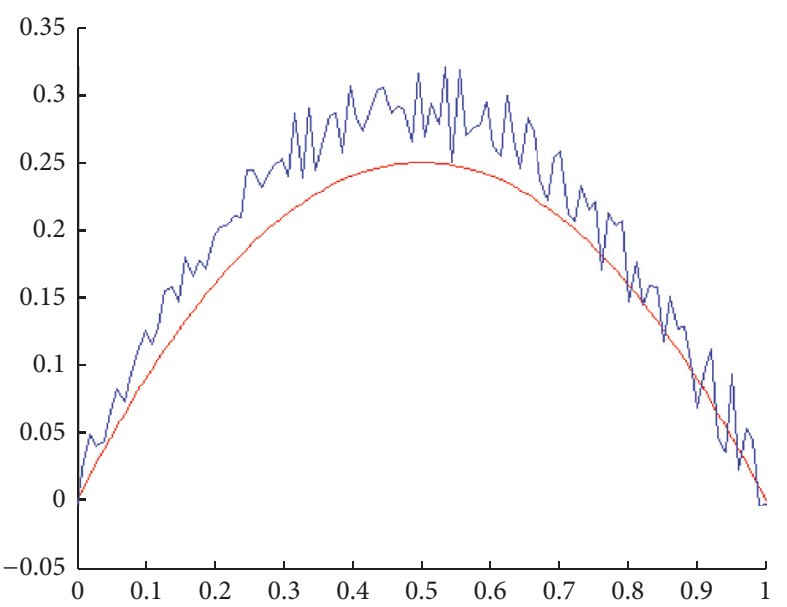

FIGURE 16: Initial temperature in err $=20 \%\left\|\psi_{0}^{\text {exact }}\right\|_{2}$ case. This figure shows that we cannot rebuild the true state.

the optimal solution [21-24]. The basic structure of a GA is the following:

(1) Initialize a population of individuals;

(2) Evaluate each individual in the population;

(3) While the stop criterion is not reached do \{

(4) Select individuals for the next population;

(5) Apply genetic operators (crossover, mutation) to produce new individuals;

(6) Evaluate the new individuals;

\}

(7) return the best individual.

The hybrid methods combine principles from genetic algorithms and other optimization methods. In this approach, we will combine the genetic algorithm with method descent (steepest descent algorithm (FP)).

We assume that we have a partial knowledge of background state $\psi^{b}$ at certain points $\left(x_{i}\right)_{i \in I}, I \subset\{1,2, \ldots, N+1\}$.

We assume the individual is a vector $\psi_{0}$; the population is a set of individuals.

The initialization of individual is as follows:

$$
\begin{aligned}
& \text { for } i=1 \text { to } N+1 \\
& \text { if } i \in I \\
& \quad \psi_{0}\left(x_{i}\right) \text { is chosen in the vicinity of } \psi^{b}\left(x_{i}\right) \\
& \text { else } \\
& \quad \psi_{0}\left(x_{i}\right) \text { is chosen randomly } \\
& \text { end if } \\
& \text { end for. }
\end{aligned}
$$

Starting by initial population, we apply genetic operators (crossover, mutation) to produce a new population in which 


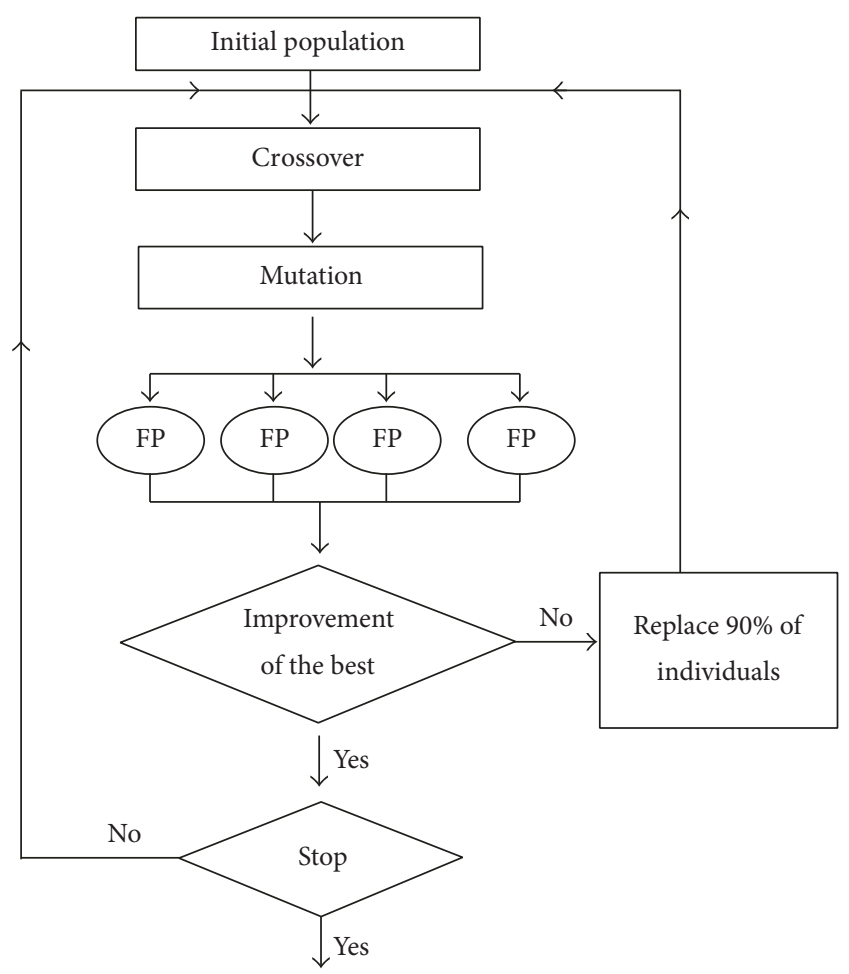

FIGURE 17: Hybrid algorithm.

each individual is an initial point for the descent method (FP). When a specific number of generations is reached without improvement of the best individual, only the fittest individuals (e.g., the first $10 \%$ fittest individuals in the population) survive. The remaining die and their place is occupied by new individuals with new genetic ( $45 \%$ are chosen randomly; the other $45 \%$ are chosen as (96)). At each generation we keep the best. The algorithm ends when $\left|J\left(\psi_{0}\right)\right|<\mu$ or generation $\geqslant$ Maxgen, where $\mu$ is a given precision (see Figure 17).

The main steps for descent method (FP) at each iteration are the following:

(i) Calculate $\psi^{k}$ solution of (6) with initial condition $\psi_{0}$.

(ii) Calculate $P^{k}$ solution of (76).

(iii) Calculate the descent direction $d_{k}=-\nabla J\left(\psi_{0}\right)$.

(iv) Find $t_{k}=\operatorname{argmin}_{t>0} J\left(\psi_{0}+t d_{k}\right)$.

(v) Update the variable $\psi_{0}=\psi_{0}+t_{k} d_{k}$.

The algorithm ends when $\left|J\left(\psi_{0}\right)\right|<\mu$, where $\mu$ is a given small precision.

$t_{k}$ is chosen by the inaccurate linear search by Rule Armijo-Goldstein as follows:

$$
\begin{aligned}
& \text { let } \alpha_{i}, \beta \in[0,1[\text { and } \alpha>0 . \\
& \text { if } J\left(\psi_{0}^{k}+\alpha_{i} d_{k}\right) \leqslant J\left(\psi_{0}^{k}\right)+\beta \alpha_{i} d_{k}^{T} d_{k} \\
& t_{k}=\alpha_{i} \text { and stop } \\
& \text { if not } \\
& \alpha_{i}=\alpha \alpha_{i} .
\end{aligned}
$$

TABLE 1: Results on the Tikhonov approach. Comparison between different values of regularizing coefficient $\varepsilon$. The smallest value of $J$ is reached when $\varepsilon=10^{-06}$.

\begin{tabular}{lcc}
\hline$\varepsilon$ & Minimum value of $J$ & Elapsed time (seconds) \\
\hline $10^{-08}$ & $1.106317 \cdot 10^{-02}$ & 8.38 \\
$10^{-07}$ & $1.092014 \cdot 10^{-02}$ & 24.47 \\
$10^{-06}$ & $6.630517 \cdot 10^{-03}$ & 23.11 \\
$10^{-05}$ & $7.752620 \cdot 10^{-03}$ & 21.50 \\
$10^{-04}$ & $7.857129 \cdot 10^{-03}$ & 22.64 \\
$10^{-03}$ & $8.510799 \cdot 10^{-03}$ & 18.95 \\
$10^{-02}$ & $8.733989 \cdot 10^{-03}$ & 15.01 \\
$10^{-01}$ & $1.018406 \cdot 10^{-02}$ & 17.33 \\
1 & $1.552344 \cdot 10^{-02}$ & 6.04 \\
\hline
\end{tabular}

TABLE 2: Results on the hybrid method.

\begin{tabular}{lc}
\hline Minimum value of $J$ & Elapsed time \\
\hline $6.581908 \cdot 10^{-03}$ & $1 \mathrm{~min}$ \\
$5.850810 \cdot 10^{-03}$ & $2 \mathrm{~min}$ \\
$3.362100 \cdot 10^{-04}$ & $7 \mathrm{~min}$ \\
$1.071378 \cdot 10^{-04}$ & $11 \mathrm{~min}$ \\
$8.739839 \cdot 10^{-05}$ & $23 \mathrm{~min}$ \\
$5.958016 \cdot 10^{-05}$ & $6 \mathrm{~h} 43 \mathrm{~min}$ \\
$6.175260 \cdot 10^{-06}$ & $11 \mathrm{~h} 20 \mathrm{~min}$ \\
\hline
\end{tabular}

Consider we know $20 \%$ of values of background state $\left(\psi^{b}\right)$, in this test we try to build the solution with the hybrid method.

In the figures below, the observed function is drawn in red and built function in blue.

Let $N$ be number of points in space and $M$ number of points in time.

6.2.1. The Noncoercive Case. Let $\alpha=1 / 2, \lambda=0$ and parameters $N=100, M=100$, number of individuals $=30$, and number of generations $=2000$.

The test with simple descent gives Figure 18.

The test with genetic algorithm gives Figure 19.

Now we turn the hybrid algorithm. This gives Figure 20.

6.2.2. Subcritical Potential Case. Let $\alpha=1 / 2, \lambda=-(1-\alpha)^{2} / 4$, $\beta=3 / 4$ and parameters $N=100, M=100$, number of individuals $=30$, and number of generations $=2000$.

The test with simple descent gives Figure 21.

The test with genetic algorithm gives Figure 22 .

Now we turn the hybrid algorithm. This gives Figure 23.

6.3. Comparison between Hybrid Approach and Tikhonov Approach. Here, we assume that we know $20 \%$ of values of background state $\left(\psi^{b}\right)$.

(i) Noncoercive Case. see Tables 1 and 2.

The minimum value of $J$ reached by the Tikhonov algorithm was $6.630517 \cdot 10^{-03}$, whereas with the hybrid 

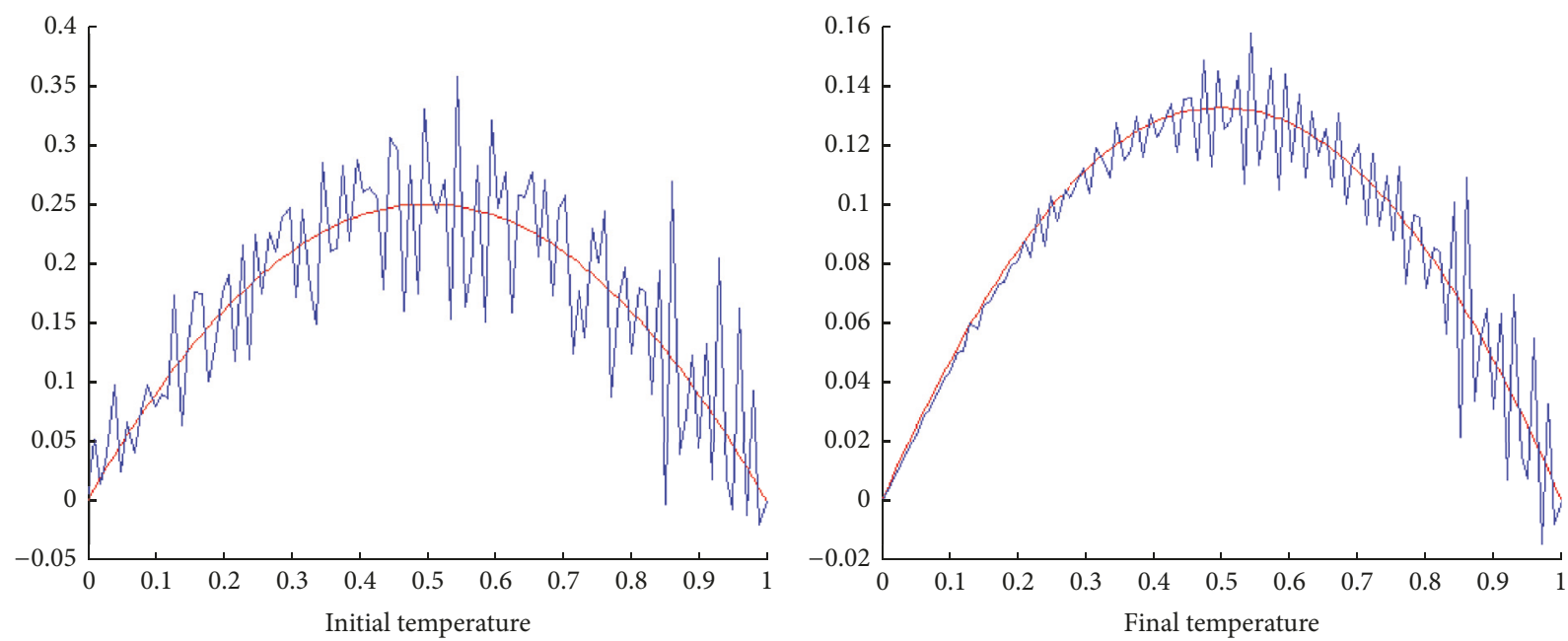

FIgURE 18: This figure shows that we cannot rebuild the solution.
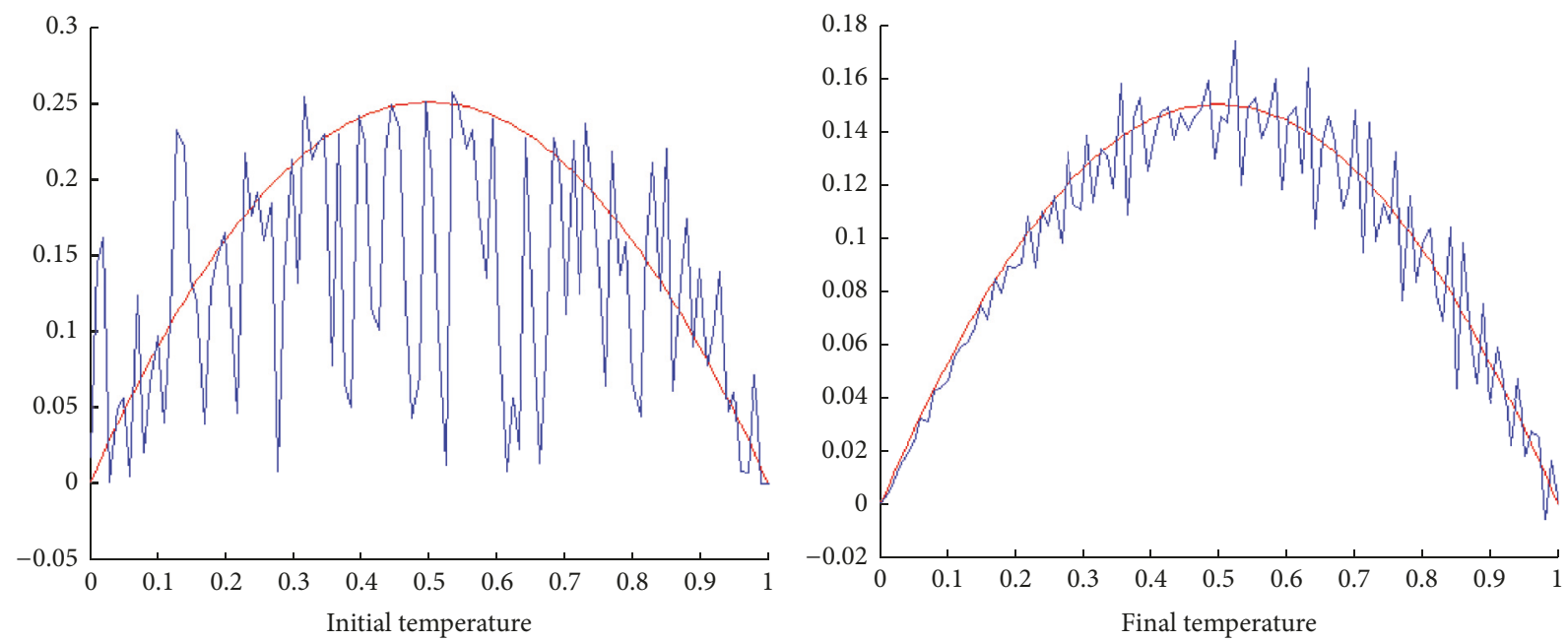

FIgURE 19: This figure shows that we cannot rebuild the solution.
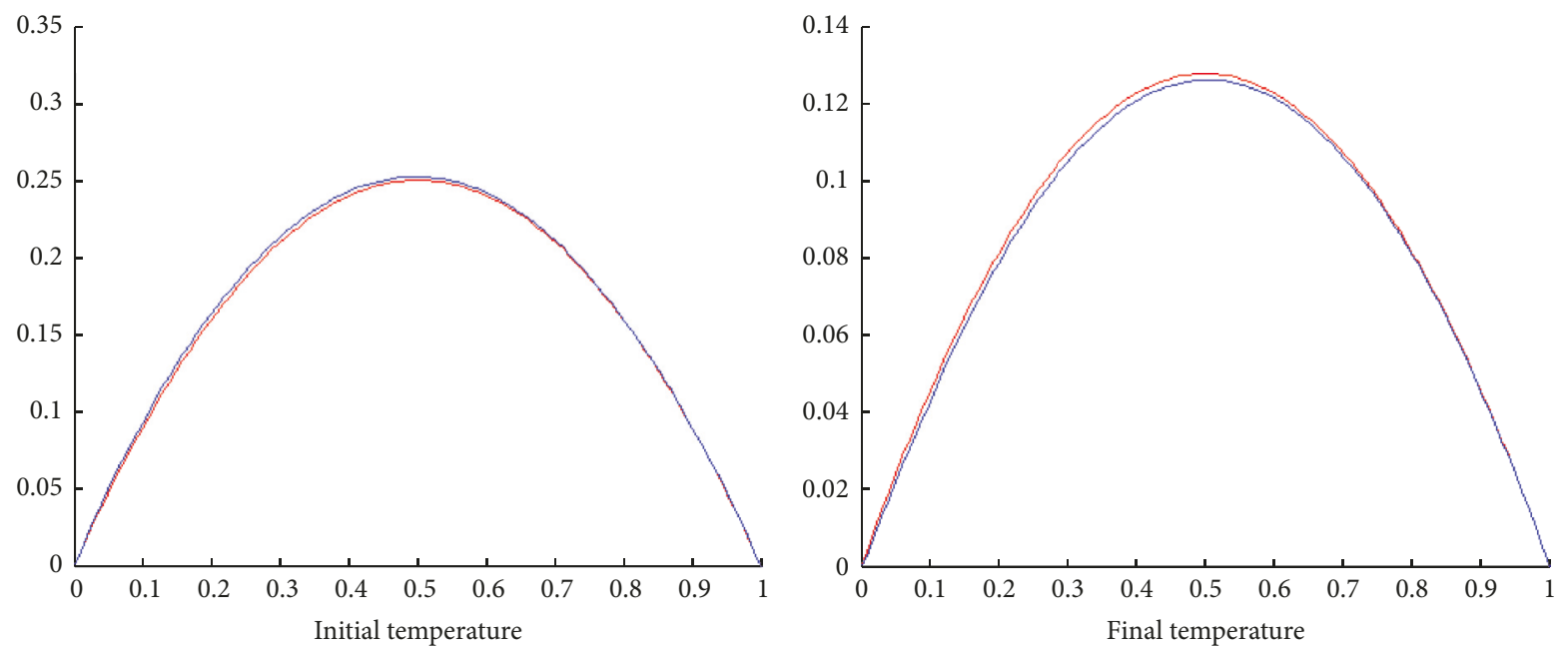

FIGURE 20: This figure shows that we can rebuild the solution. 

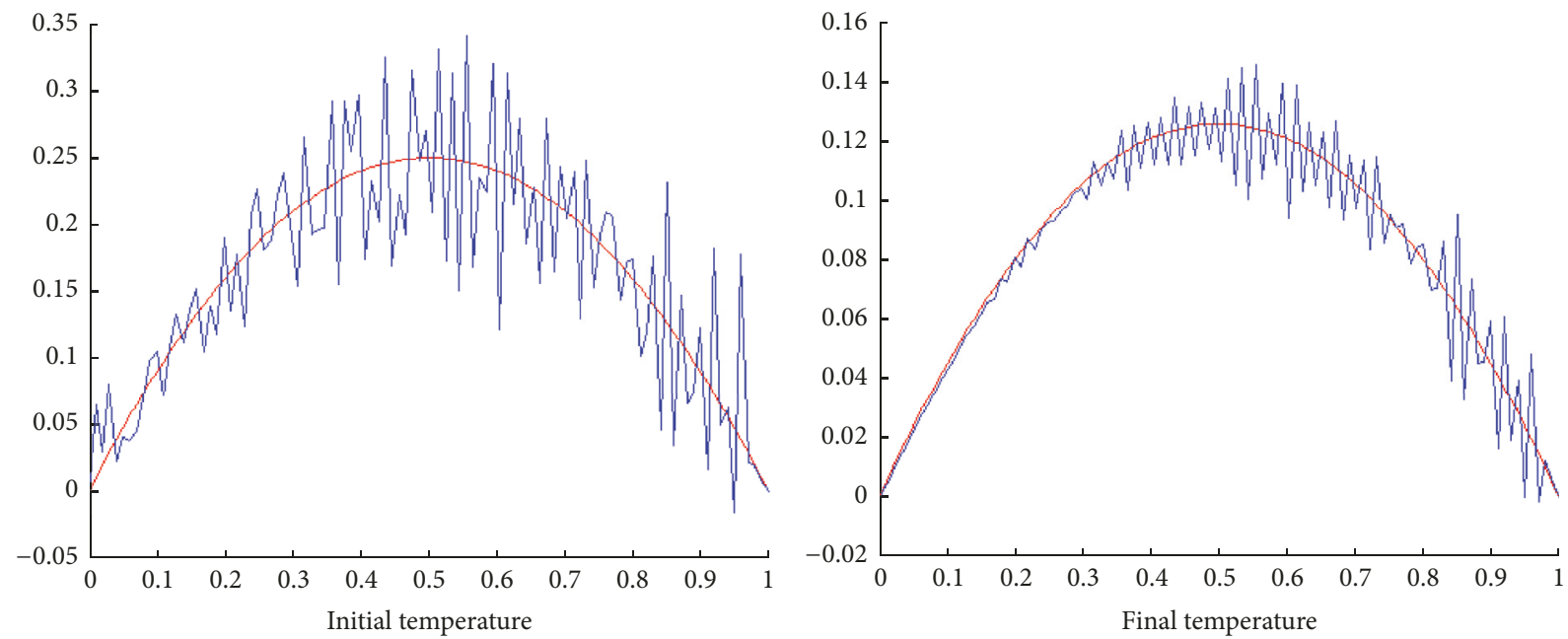

FIgURE 21: This figure shows that we cannot rebuild the solution.
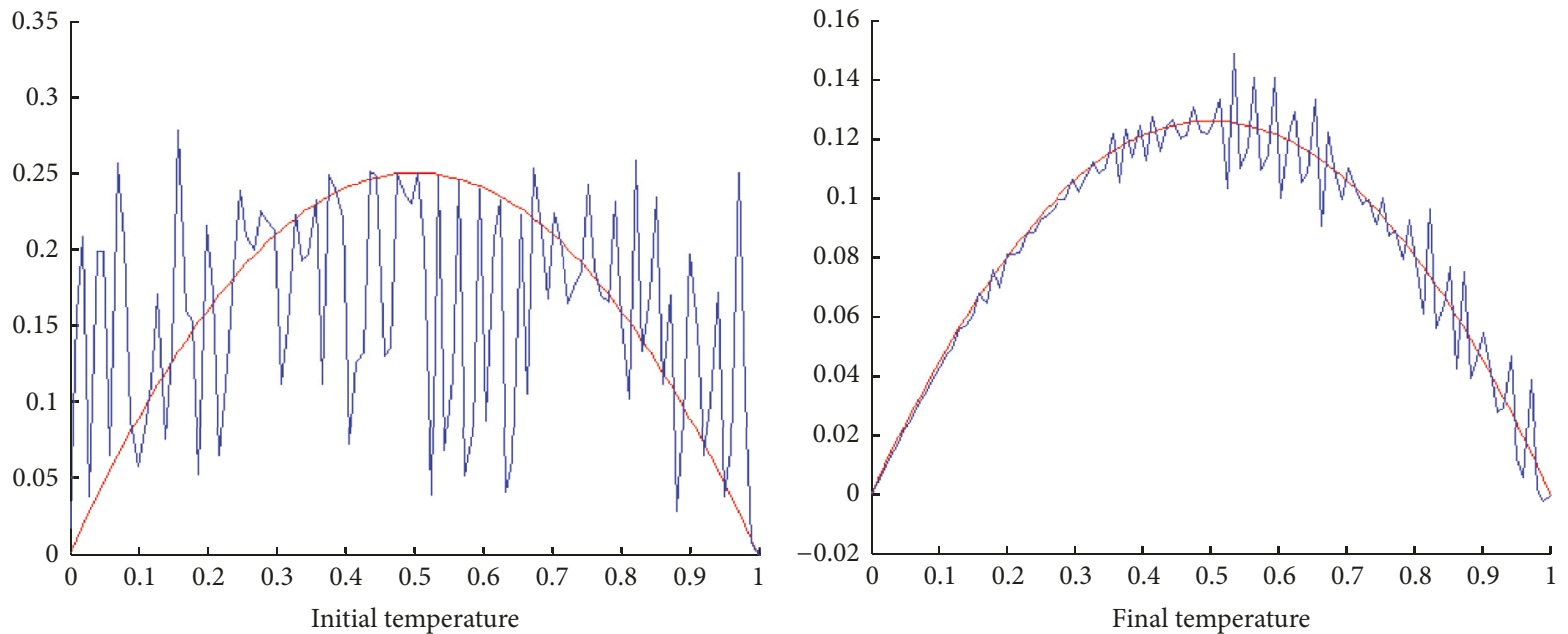

FIgURE 22: This figure shows that we cannot rebuild the solution.
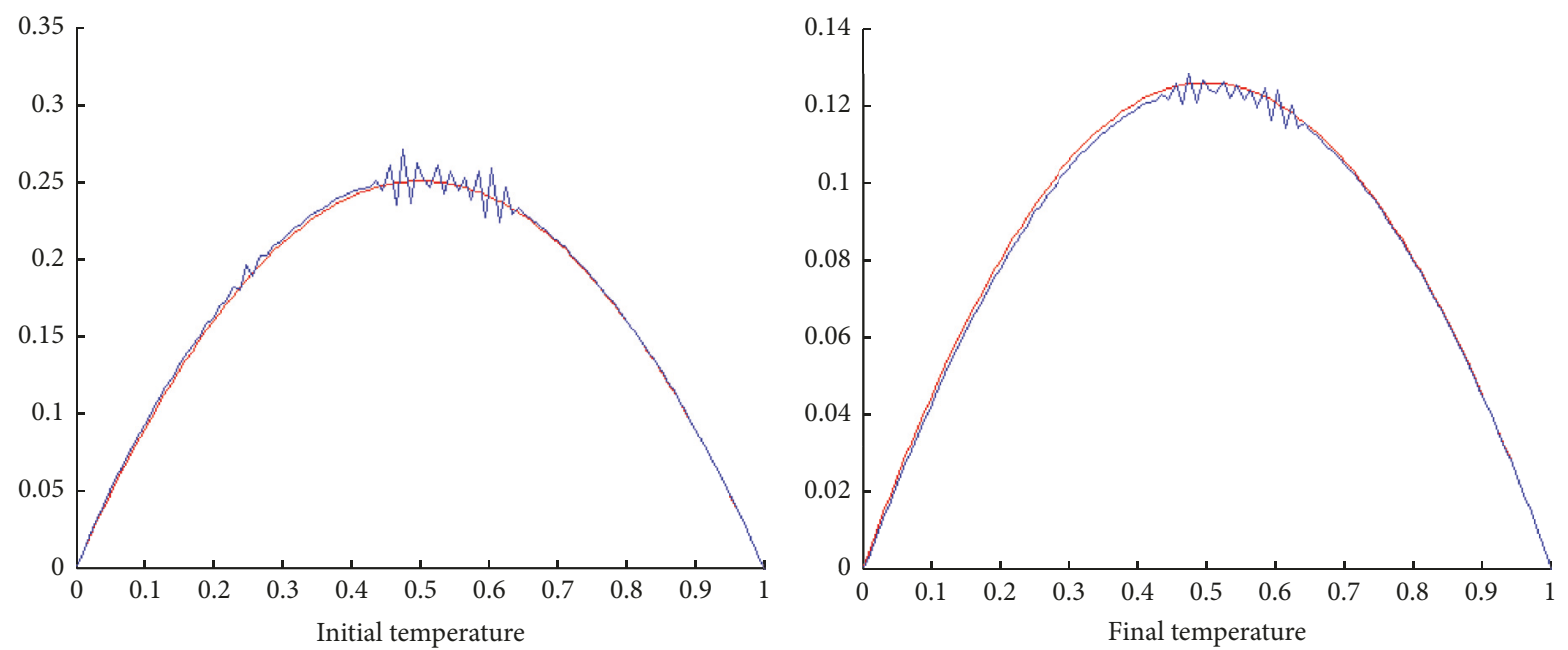

FIGURE 23: This figure shows that we can rebuild the solution. 
TABLE 3: Results on the Tikhonov aporoach. Comparison between different values of regularizing coefficient $\varepsilon$. The smallest value of $J$ is reached when $\varepsilon=10^{-03}$.

\begin{tabular}{lcc}
\hline$\varepsilon$ & Minimum value of $J$ & Elapsed time (seconds) \\
\hline $10^{-08}$ & $1.113538 \cdot 10^{-02}$ & 10.91 \\
$10^{-07}$ & $1.188092 \cdot 10^{-02}$ & 8.68 \\
$10^{-06}$ & $1.099187 \cdot 10^{-02}$ & 8.75 \\
$10^{-05}$ & $1.267204 \cdot 10^{-02}$ & 7.91 \\
$10^{-04}$ & $9.648060 \cdot 10^{-03}$ & 11.17 \\
$10^{-03}$ & $7.320995 \cdot 10^{-03}$ & 11.61 \\
$10^{-02}$ & $9.188603 \cdot 10^{-03}$ & 10.20 \\
$10^{-01}$ & $9.799159 \cdot 10^{-03}$ & 9.57 \\
1 & $1.042463 \cdot 10^{-02}$ & 9.66 \\
\hline
\end{tabular}

TABLE 4: Results on the hybrid method.

\begin{tabular}{lc}
\hline Minimum value of $J$ & Elapsed time \\
\hline $7.605018 \cdot 10^{-03}$ & $1 \mathrm{~min}$ \\
$7.505279 \cdot 10^{-03}$ & $2 \mathrm{~min}$ \\
$1.762564 \cdot 10^{-03}$ & $4 \mathrm{~min}$ \\
$9.407809 \cdot 10^{-04}$ & $43 \mathrm{~min}$ \\
$2.981666 \cdot 10^{-05}$ & $1 \mathrm{~h} 56 \mathrm{~min}$ \\
$1.378356 \cdot 10^{-05}$ & $6 \mathrm{~h} 43 \mathrm{~min}$ \\
$8.203546 \cdot 10^{-06}$ & $13 \mathrm{~h} 40 \mathrm{~min}$ \\
\hline
\end{tabular}

algorithm it was possible to reach the value $6.175260 \cdot 10^{-06}$ in $11 \mathrm{~h}$ and $20 \mathrm{~min}$ with knowledge of $20 \%$ of $\psi^{b}$; if we take more than $20 \%$, we got less than elapsed time.

(ii) Subcritical Potential Case. see Tables 3 and 4.

The minimum value of $J$ reached by the Tikhonov algorithm was $7.320995 \cdot 10^{-03}$, whereas with the hybrid algorithm it was possible to reach the value $8.203546 \cdot 10^{-06}$ in $13 \mathrm{~h}$ and $40 \mathrm{~min}$ with knowledge of $20 \%$ of $\psi^{b}$; if we take more than $20 \%$, we got less than elapsed time.

\section{Conclusion}

In this paper, we have presented the regularization method and the hybrid method which are applied to determine an initial state from the point of measurements of parabolic degenerate/singular problem. These methods have proven efficiency to rebuild the solution. The proposed reconstruction algorithms are easily implanted.

The elapsed time of the hybrid method is long enough. To reduce it, in our coming work we will use the multiprogramming to run two approaches of parallelism.

\section{Conflicts of Interest}

The authors declare that there are no conflicts of interest regarding the publication of this paper.

\section{References}

[1] V. Isakov, Inverse Problems for Partial Differential Equations, Springer, New York, NY, USA, 1998.

[2] A. Kirsch, An Introduction to the Mathematical Theory of Inverse Problems, Springer, New York, NY, USA, 1999.

[3] J.-M. Buchot and J.-P. Raymond, "A linearized model for boundary layer equations, Optimal control of complex structures (Oberwolfach, 2000)," in Internat. Ser. Numer. Math, vol. 139, pp. 31-42, Birkhäuser, Basel, Swizerland, 2002.

[4] K. Beauchard and E. Zuazua, "Some controllability results for the 2D Kolmogorov equation," Annales de l'Institut Henri Poincare (C) Non Linear Analysis, vol. 26, no. 5, pp. 1793-1815, 2009.

[5] N. Shimakura, Partial differential operators of elliptic type, vol. 99 of Translations of Mathematical Monographs, American Mathematical Society, Providence, RI, USA, 1992.

[6] W. H. Fleming and M. Viot, "Some measure-valued Markov processes in population genetics theory," Indiana University Mathematics Journal, vol. 28, no. 5, pp. 817-843, 1979.

[7] H. Emamirad, G. R. Goldstein, and J. A. Goldstein, "Chaotic solution for the Black-Scholes equation," Proceedings of the American Mathematical Society, vol. 140, no. 6, pp. 2043-2052, 2012.

[8] G. Fragnelli and D. Mugnai, "Carleman estimates for singular parabolic equations with interior degeneracy and non smooth coefficients," Advances in Nonlinear Analysis, 2016.

[9] F. Bourquin and A. Nassiopoulos, "Assimilation thermique 1D par méthode adjointe libérée," in Problèmes Inverses, Collection Recherche du LCPC, 2006.

[10] T. Min, B. Geng, and J. Ren, "Inverse estimation of the initial condition for the heat equation," International Journal of Pure and Applied Mathematics, vol. 82, no. 4, pp. 581-593, 2013.

[11] L. B. L. Santos, L. D. Chiwiacowsky, and H. F. Campos-Velho, "Genetic algorithm and variational method to identify initial conditions: worked example in hyperbolic heat transfer," TEMA. Tendências em Matemática Aplicada e Computacional, vol. 14, no. 2, pp. 265-276, 2013.

[12] M. Bonnet, Ecole Centrale de Paris Mention Matière, Structures, Fluides, Rayonnement Spécialité Dynamique des Structures et Systèmes Couplés [M.S. thesis], 2008, bonnet@lms.polytechnique.fr.

[13] F. Jens, Generalized Tikhonov regularization, Basic theory and comprehensive results on convergence rates [Dissertation], Fakultät für Mathematik Technische Universität Chemnitz, Chemnitz, 2011.

[14] F. Alabau-Boussouira, P. Cannarsa, and G. Fragnelli, “Carleman estimates for degenerate parabolic operators with applications to null controllability," Journal of Evolution Equations, vol. 6, no. 2, pp. 161-204, 2006.

[15] E. M. Ait Ben Hassi, F. Ammar Khodja, A. Hajjaj, and L. Maniar, "Carleman estimates and null controllability of coupled degenerate systems," Evolution Equations and Control Theory, vol. 2, no. 3, pp. 441-459, 2013.

[16] P. Cannarsa and G. Fragnelli, "Null controllability of semilinear degenerate parabolic equations in bounded domains," Electronic Journal of Differential Equations, vol. 136, pp. 1-20, 2006

[17] P. Cannarsa, P. Martinez, and J. Vancostenoble, "Carleman estimates for a class of degenerate parabolic operators," SIAM Journal on Control and Optimization, vol. 47, no. 1, article no. 119, 2008. 
[18] P. Cannarsa, P. Martinez, and J. Vancostenoble, "Null controllability of degenerate heat equations," Advances in Differential Equations, vol. 10, no. 2, pp. 153-190, 2005.

[19] J. Vancostenoble, "Improved Hardy-Poincaré inequalities and sharp Carleman estimates for degenerate/singular parabolic problems," Discrete and Continuous Dynamical Systems, vol. 4, no. 3, Article ID 761790, 2011.

[20] E. M. Ait Ben Hassi, F. Ammar Khodja, A. Hajjaj, and L. Maniar, "Null controllability of degenerate cascade systems," Journal of Evolution Equations and Control Theory, vol. 2, no. 3, pp. 441459, 2013.

[21] Z. Michalewicz, Genetic Algorithms + Data Structures = Evolution Programs, Springer, New York, NY, USA, 1996.

[22] D. E. Goldberg, Genetic Algorithms in Search, Optimisation and Machine Learning, 1989.

[23] J. H. Holland, Adaptation in Natural and Artificial Systems, University of Michigan Press, Oxford, UK, 1975.

[24] J. R. Koza, Genetic Programming, MIT Press, 1992. 


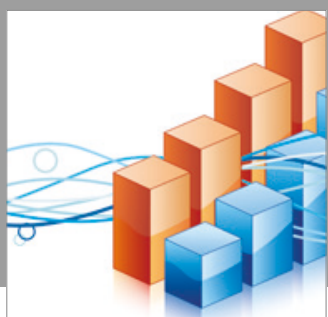

Advances in

Operations Research

vatersals

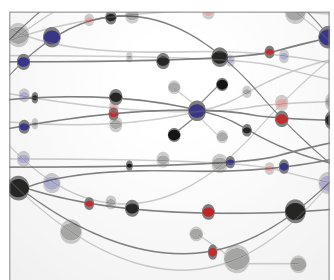

\section{The Scientific} World Journal
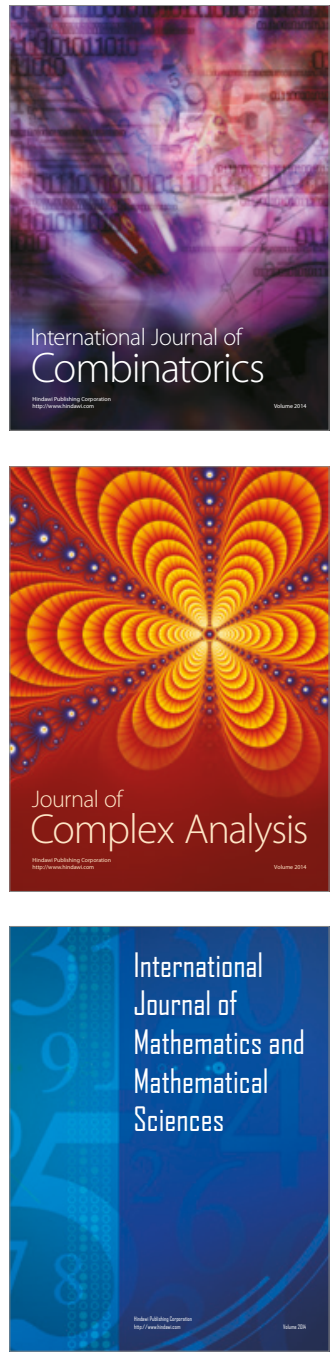
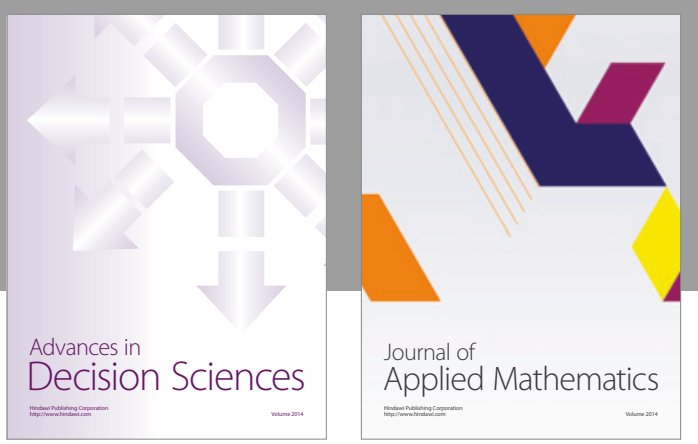

Algebra

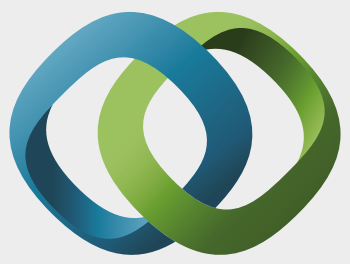

\section{Hindawi}

Submit your manuscripts at

https://www.hindawi.com
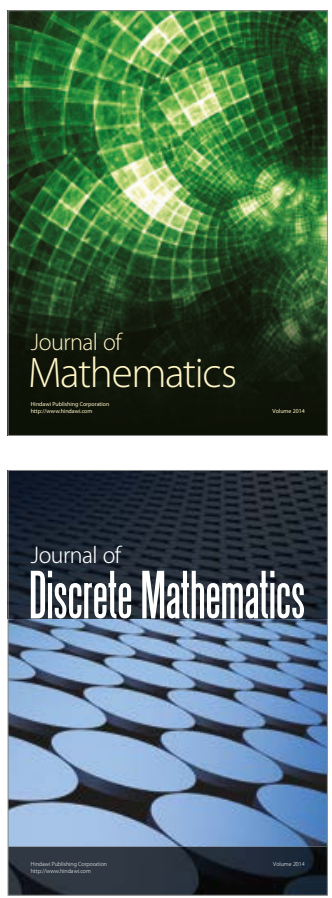

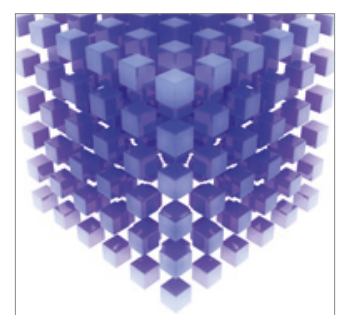

Mathematical Problems in Engineering
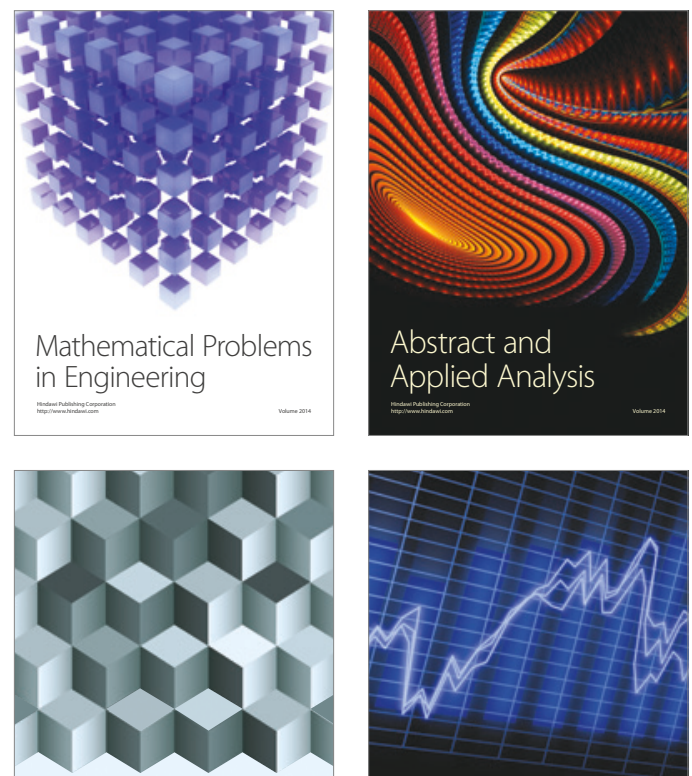

Journal of

Function Spaces

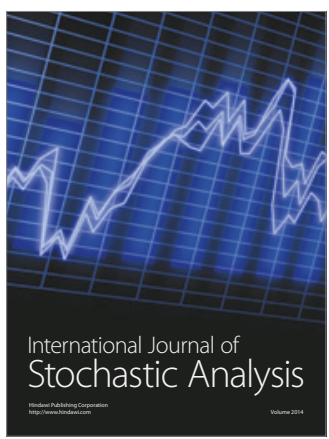

Probability and Statistics
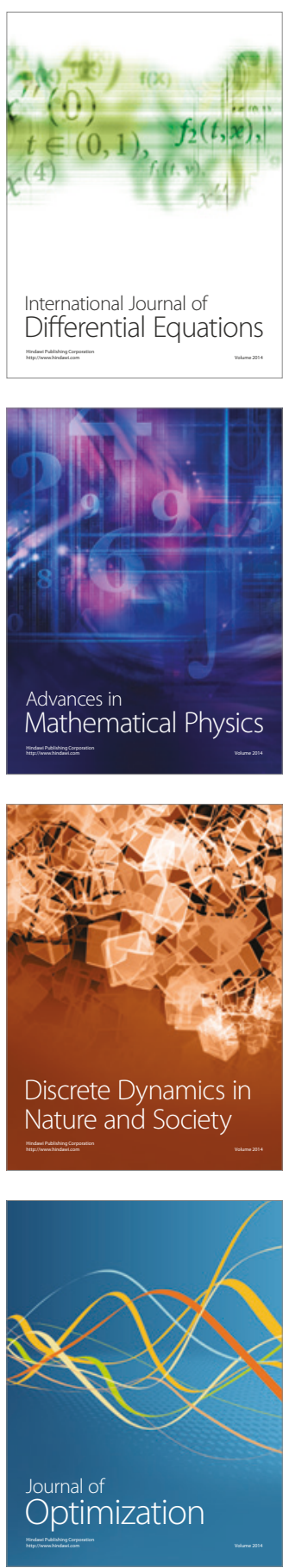